\title{
A highly selective synthesis of dialkenyl sulfones via cross-metathesis based desymmetrization of divinyl sulfone
}

\author{
Supplementary Information \\ Michal Bieniek, ${ }^{\text {a }}$ Dariusz Kołoda, ${ }^{\text {a,b }}$ and Karol Grela ${ }^{\mathrm{a}, *}$ \\ ${ }^{a}$ Institute of Organic Chemistry, Polish Academy of Sciences \\ Kasprzaka 44/52, 01-224 Warsaw, Poland \\ ${ }^{b}$ Faculty of Chemistry, Warsaw University of Technology (Politechnika), Noakowskiego 3, 00-664 \\ Warsaw, Poland \\ grela@icho.edu.pl
}

\section{General}

Unless otherwise noted, all reactions were carried out under Ar in pre-dried glassware using Schlenk techniques. The solvents were dried by distillation over the following drying agents and were transferred under argon: THF (K/benzophenone), toluene (Na), n-pentane, $n$-hexane, $\mathrm{CH}_{2} \mathrm{Cl}_{2}\left(\mathrm{CaH}_{2}\right)$, $\mathrm{Et}_{2} \mathrm{O}\left(\mathrm{LiAlH}_{4}\right)$. Flash column chromatography: Merck silica gel 60 (230-400 mesh). NMR: Spectra were recorded on Bruker AVANCE 500, Varian Gemini 200 and 400 spectrometers in $\mathrm{CDCl}_{3}$; chemical shifts $(\delta)$ are given in ppm relative to TMS, coupling constants $(J)$ in Hz. IR: Perkin-Elmer Spectrum 2000 FT-IR, wavenumbers in $\mathrm{cm}^{-1}$. MS (EI, LSIMS): AMD 604 Intectra GmbH. MS (ESI): Mariner Perseptive Biosystems, Inc. HPLC-MS (API): HP 1100 series with PE SCIEX API 365 turbo-ion spray and Nucleosil 1000-C18 column. Micro-analyses were provided by Institute of Organic Chemistry, PAS, Warsaw. All commercially available substrates were used as received.

\section{Exprimental part}

\subsection{Representative procedure for $\mathrm{CM}$ of divinyl sulfone}

To a mixture of alkene $(2.0 \mathrm{mmol})$ and divinylsulfone $(6.0 \mathrm{mmol})$ in $\mathrm{CH}_{2} \mathrm{Cl}_{2}(100 \mathrm{~mL}, c=0.02 \mathrm{M})$ was added a Ru-catalyst $\mathbf{1}$ or $\mathbf{4}$ as a solid $(5 \mathrm{~mol} \%)$. The resulted mixture was stirred at reflux for 16-24 $\mathrm{h}$ under Ar. The solvent was removed under reduced pressure. The crude product was purified by flash chromatography using $c$-hexan/ethyl acetate as eluent (Merck silica gel 60, 230-400 mesh).

\section{(1E)-3-phenylprop-1-en-1-yl vinyl sulfone (7b)}

Yellow oil (84 \%). IR (film): $\nu$ 3104, 3057, 3030, 2921, 2256, 1957, 1734, 1630, 1604, 1496, 1454, $1384,1314,1127,1029,976,851,792,700,645,585,486 \mathrm{~cm}^{-1} \cdot{ }^{1} \mathrm{H}$ NMR $\left(400 \mathrm{MHz}, \mathrm{CDCl}_{3}\right) \delta$ $3.60(\mathrm{dd}, J=6.4,1.8 \mathrm{~Hz}, 2 \mathrm{H}), 6.05(\mathrm{~d}, J=9.9 \mathrm{~Hz}, 1 \mathrm{H}), 6.16(\mathrm{dt}, J=15.1,1.8 \mathrm{~Hz}, 1 \mathrm{H}), 6.37$ $(\mathrm{d}, J=16.5 \mathrm{~Hz}, 1 \mathrm{H}), 6.56(\mathrm{dd}, J=16.5,9.9 \mathrm{~Hz}, 1 \mathrm{H}), 7.11(\mathrm{dt}, J=15.1,6.4 \mathrm{~Hz}, 1 \mathrm{H}), 7.14-7.18$ (m, 2H), 7.24-7.30 (m, 1H), 7.31-7.36 (m, 2H) ppm. ${ }^{13} \mathrm{C}$ NMR (100 MHz, $\left.\mathrm{CDCl}_{3}\right) \delta 37.7,127.1$, 128.7, 128.8, 128.9, 129.7, 136.0, 137.4, 147.6. MS (EI) $\mathrm{m} / \mathrm{z}$ (rel intensity) $208\left(18, \mathbf{M}^{+\cdot}\right.$ ), 163 (5), 142 (8), 118 (10), 117 (100), 116 (41), 115 (71), 91 (34), 65 (13), 51 (5), 39 (8). HRMS (EI) calcd for $\mathrm{M}^{+\cdot}\left(\mathrm{C}_{11} \mathrm{H}_{12} \mathrm{O}_{2} \mathrm{~S}\right)$ : 208.05580; found: 208.05669. Anal. calcd for $\mathrm{C}_{11} \mathrm{H}_{12} \mathrm{O}_{2} \mathrm{~S}$ (208.28): C 63.43, H 5.81, S 15.39; found C 63.28, H 6.03, S 15.21.

\section{(1E)-1-(Vinylsulfonyl)hept-1-ene (7c)}

Yellow oil (51 \%). IR (film): $\nu$ 3055, 3020, 2961, 2928, 2859, 1627, 1462, 1416, 1381, 1316, 1261, 1129, 1016, 818, 720, 647, 555, $499 \mathrm{~cm}^{-1} .{ }^{1} \mathrm{H}$ NMR $\left(400 \mathrm{MHz}, \mathrm{CDCl}_{3}\right) \delta 0.90(\mathrm{t}, J=7.0 \mathrm{~Hz}$, $3 \mathrm{H}), 1.27-1.35(\mathrm{~m}, 4 \mathrm{H}), 1.44-1.54(\mathrm{~m}, 2 \mathrm{H}), 2.23-2.32(\mathrm{~m}, 2 \mathrm{H}), 6.05(\mathrm{~d}, J=9.8 \mathrm{~Hz}, 1 \mathrm{H}), 6.23(\mathrm{dt}$, $J=15.1,1.6 \mathrm{~Hz}, 1 \mathrm{H}), 6.39(\mathrm{~d}, J=16.6 \mathrm{~Hz}, 1 \mathrm{H}), 6.59(\mathrm{dd}, J=16.6,9.8 \mathrm{~Hz}, 1 \mathrm{H}), 6.95(\mathrm{dt}, J=$ 
15.1, $6.8 \mathrm{~Hz}, 1 \mathrm{H}) \mathrm{ppm} .{ }^{13} \mathrm{C} \mathrm{NMR}\left(100 \mathrm{MHz}, \mathrm{CDCl}_{3}\right) \delta 13.9,22.3,27.2,31.1,31.6,128.3,128.5$, 137.7, 149.5. MS (EI) $\mathrm{m} / z$ (rel intensity) 145 (4), 133 (6), 119 (32), 96 (17), 93 (14), 81 (70), 75 (13), 69 (12), 67 (54), 57 (10), 55 (100), 53 (15), 43 (21), 41 (79), 39 (31). HRMS (ESI) calcd for $[\mathrm{M}+\mathrm{Na}]^{+}\left(\mathrm{C}_{9} \mathrm{H}_{16} \mathrm{O}_{2} \mathrm{SNa}\right): 211.0763$; found: 211.0774 .

(1E)-1-(Vinylsulfonyl)nonadec-1-ene (7d)

Colourless powder (70 \%). IR (KBr): $\nu$ 3066, 3052, 2955, 2918, 2849, 1629, 1472, 1463, 1384, 1305, 1124, 967, 847, 824, 805, 777, 759, 730, 720, 645, 554, 504, $492 \mathrm{~cm}^{-1} .{ }^{1} \mathrm{H}$ NMR (400 MHz, $\left.\mathrm{CDCl}_{3}\right) \delta 0.87(\mathrm{t}, J=7.0 \mathrm{~Hz}, 3 \mathrm{H}), 1.20-1.34(\mathrm{~m}, 28 \mathrm{H}), 1.42-1.52(\mathrm{~m}, 2 \mathrm{H}), 2.22-2.30(\mathrm{~m}, 2 \mathrm{H})$, $6.04(\mathrm{~d}, J=9.8 \mathrm{~Hz}, 1 \mathrm{H}), 6.22(\mathrm{dt}, J=15.2,1.5 \mathrm{~Hz}, 1 \mathrm{H}), 6.38(\mathrm{~d}, J=16.6 \mathrm{~Hz}, 1 \mathrm{H}), 6.57(\mathrm{dd}, J$ $=16.6,9.8 \mathrm{~Hz}, 1 \mathrm{H}), 6.94(\mathrm{dt}, J=15.2,6.8 \mathrm{~Hz}, 1 \mathrm{H}) \mathrm{ppm} .{ }^{13} \mathrm{C} \mathrm{NMR}\left(100 \mathrm{MHz}, \mathrm{CDCl}_{3}\right) \delta 14.1$, $22.7,27.5,29.0,29.2,29.3,29.4,29.5,29.6,29.7,31.7,31.9,128.3,128.5,137.7,149.6$. MS (EI) $\mathrm{m} / \mathrm{z}$ (rel intensity) 339 (14), 321 (5), 151 (6), 145 (13), 123 (30), 109 (55), 95 (85), 81 (100), 69 (76), 57 (73), 55 (79), 53 (15), 43 (79), 41 (61). HRMS (ESI) calcd for $[\mathrm{M}+\mathrm{Na}]^{+}\left(\mathrm{C}_{21} \mathrm{H}_{40} \mathrm{O}_{2} \mathrm{SNa}\right)$ : 379.2641; found: 379.2650. Anal. calcd for $\mathrm{C}_{21} \mathrm{H}_{40} \mathrm{O}_{2} \mathrm{~S}$ (356.62): C 70.73, H 11.31, S 8.99; found C $70.65, \mathrm{H} 11.15, \mathrm{~S} 8.82$.

\section{Tert-butyl(dimethyl)[(5E)-6-(vinylsulfonyl)hex-5-en-1-yl]oxysilane (7e)}

Yellow oil (69 \%). IR (film): $\nu$ 3055, 2953, 2930, 2886, 2858, 1628, 1472, 1463, 1386, 1361, 1320, $1255,1133,1099,1006,974,835,776,719,646,497 \mathrm{~cm}^{-1} .{ }^{1} \mathrm{H}$ NMR $\left(500 \mathrm{MHz}, \mathrm{CDCl}_{3}\right) \delta 0.00$ $(\mathrm{s}, 6 \mathrm{H}), 0.83(\mathrm{~s}, 9 \mathrm{H}), 1.45-1.54(\mathrm{~m}, 4 \mathrm{H}), 2.21-2.29(\mathrm{~m}, 2 \mathrm{H}), 5.99(\mathrm{~d}, J=9.9 \mathrm{~Hz}, 1 \mathrm{H}), 6.20(\mathrm{~d}, J$ $=15.2 \mathrm{~Hz}, 1 \mathrm{H}), 6.31(\mathrm{~d}, J=16.6 \mathrm{~Hz}, 1 \mathrm{H}), 6.53(\mathrm{dd}, J=16.6,9.9 \mathrm{~Hz}, 1 \mathrm{H}), 6.88(\mathrm{dt}, J=15.2$, $6.8 \mathrm{~Hz}, 1 \mathrm{H}) \mathrm{ppm} .{ }^{13} \mathrm{C}$ NMR $\left(125 \mathrm{MHz}, \mathrm{CDCl}_{3}\right) \delta-5.5,18.1,23.9,25.8,31.2,31.8,62.3,128.1$, 128.7, 137.7, 149.0. MS (EI) m/z (rel intensity) 289 (3), 249 (10), 248 (17), 247 (100), 149 (22), 85 (42), 81 (8), 81 (100), 75 (21), 57 (8), 41 (6). HRMS (ESI) calcd for [M+Na] ${ }^{+}\left(\mathrm{C}_{14} \mathrm{H}_{28} \mathrm{O}_{3} \mathrm{SSiNa}\right)$ : 327.1421; found: 327.1440. Anal. calcd for $\mathrm{C}_{14} \mathrm{H}_{28} \mathrm{O}_{3} \mathrm{SSi}$ (304.53): C 55.22, H 9.27, S 10.53; found C 54.96, H 9.33, S 10.47.

\section{(10E)-11-(Vinylsulfonyl)undec-10-en-1-ol (7f)}

Yellow oil (71 \%). IR (film): $\nu$ 3518, 3411, 3104, 3055, 2928, 2856, 1627, 1464, 1384, 1316, 1252, 1130, 1056, 977, 825, 722, 647, 557, 500, $433 \mathrm{~cm}^{-1} .{ }^{1} \mathrm{H}$ NMR $\left(500 \mathrm{MHz}, \mathrm{CDCl}_{3}\right) \delta$ 1.25-1.38 $(\mathrm{m}, 10 \mathrm{H}), 1.41-1.51(\mathrm{~m}, 2 \mathrm{H}), 1.52-1.60(\mathrm{~m}, 2 \mathrm{H}), 2.27(\mathrm{qd}, J=6.8,1.4 \mathrm{~Hz}, 2 \mathrm{H}), 3.63(\mathrm{t}, J=$ $6.6 \mathrm{~Hz}, 2 \mathrm{H}), 6.05(\mathrm{~d}, J=9.9 \mathrm{~Hz}, 1 \mathrm{H}), 6.23(\mathrm{dt}, J=15.1,1.4 \mathrm{~Hz}, 1 \mathrm{H}), 6.38(\mathrm{~d}, J=16.6 \mathrm{~Hz}$, $1 \mathrm{H}), 6.58(\mathrm{dd}, J=16.6,9.9 \mathrm{~Hz}, 1 \mathrm{H}), 6.94(\mathrm{dt}, J=15.1,6.8 \mathrm{~Hz}, 1 \mathrm{H}) \mathrm{ppm} .{ }^{13} \mathrm{C}$ NMR $(125 \mathrm{MHz}$, $\left.\mathrm{CDCl}_{3}\right) \delta 25.6,27.5,28.9,29.1,29.2,29.3,31.6,32.7,62.9,128.2,128.6,137.8,149.4$. MS (EI) $\mathrm{m} / \mathrm{z}$ (rel intensity) 243 (1), 213 (3), 150 (8), 145 (35), 135 (12), 132 (11), 121 (26), 119 (20), 115 (16), 109 (36), 95 (88), 81 (100), 75 (19), 69 (50), 67 (82), 55 (58), 41 (38). HRMS (ESI) calcd for $[\mathrm{M}+\mathrm{Na}]^{+} \quad\left(\mathrm{C}_{13} \mathrm{H}_{24} \mathrm{O}_{3} \mathrm{SNa}\right)$ : 283.1338; found: 283.1325. Anal. calcd for $\mathrm{C}_{13} \mathrm{H}_{24} \mathrm{O}_{3} \mathrm{~S}$ (260.40): C 59.96, H 9.29, S 12.31; found C 60.02, H 9.48, S 11.98.

\section{(1E)-10-Bromo-1-(vinylsulfonyl)dec-1-ene $(7 \mathrm{~g})$}

Pale brown oil (72 \%). IR (film): $\nu$ 3103, 3054, 3017, 2930, 2856, 1628, 1463, 1438, 1383, 1317, $1252,1131,971,825,721,645,559,500,431 \mathrm{~cm}^{-1} .{ }^{1} \mathrm{H}$ NMR $\left(500 \mathrm{MHz}, \mathrm{CDCl}_{3}\right) \delta 1.28-1.36$ $(\mathrm{m}, 6 \mathrm{H}), 1.38-1.53(\mathrm{~m}, 4 \mathrm{H}), 1.81-1.88(\mathrm{~m}, 2 \mathrm{H}), 2.27(\mathrm{qd}, J=6.8,1.6 \mathrm{~Hz}, 2 \mathrm{H}), 3.40(\mathrm{t}, J=6.8$ $\mathrm{Hz}, 2 \mathrm{H}), 6.05(\mathrm{~d}, J=9.9 \mathrm{~Hz}, 1 \mathrm{H}), 6.24(\mathrm{dt}, J=15.1,1.6 \mathrm{~Hz}, 1 \mathrm{H}), 6.40(\mathrm{~d}, J=16.6 \mathrm{~Hz}, 1 \mathrm{H}), 6.58$ $(\mathrm{dd}, J=16.6,9.9 \mathrm{~Hz}, 1 \mathrm{H}), 6.94(\mathrm{dt}, J=15.1,6.8 \mathrm{~Hz}, 1 \mathrm{H}) \mathrm{ppm} .{ }^{13} \mathrm{C}$ NMR $\left(125 \mathrm{MHz}, \mathrm{CDCl}_{3}\right) \delta$ 27.5, 28.0, 28.5, 28.5, 29.0, 31.6, 32.7, 33.9, 128.2, 128.7, 137.8, 149.3. HRMS (LSIM(+)) calcd for $[\mathrm{M}+\mathrm{H}]^{+}\left(\mathrm{C}_{12} \mathrm{H}_{21} \mathrm{O}_{2} \mathrm{~S}^{79} \mathrm{Br}\right)$ : 309.05239; found: 309.05091.

\section{(1E)-6-Bromo-1-(vinylsulfonyl)hex-1-ene (7h)}

Yellow oil (36 \%). IR (film): $\nu$ 3103, 3054, 2939, 2865, 1628, 1455, 1435, 1383, 1316, 1250, 1130, 971, 827, 720, 645, 559, $502 \mathrm{~cm}^{-1} .{ }^{1} \mathrm{H}$ NMR $\left(500 \mathrm{MHz}, \mathrm{CDCl}_{3}\right) \delta 1.67$ (quint, $J=7.7 \mathrm{~Hz}, 2 \mathrm{H}$ ), 1.86-1.94 (m, 2H), $2.32(\mathrm{qd}, J=6.8,1.5 \mathrm{~Hz}, 2 \mathrm{H}), 3.41(\mathrm{t}, J=6.6 \mathrm{~Hz}, 2 \mathrm{H}), 6.07(\mathrm{~d}, J=9.8 \mathrm{~Hz}$, $1 \mathrm{H}), 6.27(\mathrm{dt}, J=15.2,1.6 \mathrm{~Hz}, 1 \mathrm{H}), 6.40(\mathrm{~d}, J=16.6 \mathrm{~Hz}, 1 \mathrm{H}), 6.58(\mathrm{dd}, J=16.6,9.8 \mathrm{~Hz}, 1 \mathrm{H})$, $6.94(\mathrm{dt}, J=15.2,6.8 \mathrm{~Hz}, 1 \mathrm{H}) \mathrm{ppm} .{ }^{13} \mathrm{C} \mathrm{NMR}\left(125 \mathrm{MHz}, \mathrm{CDCl}_{3}\right) \delta 26.0,30.7,31.8,32.8,128.6$, 129.3, 137.6, 148.1. MS (EI) $\mathrm{m} / \mathrm{z}$ (rel intensity) $252\left(3, \mathrm{M}^{+\cdot}\right.$ ), 237 (44), 235 (44), 209 (10), 187 (10), 173 (25), 145 (84), 119 (21), 107 (18), 93 (15), 81 (100), 79 (28), 75 (13), 67 (12), 55 (12), 53 
(13), 41 (32), 39 (18). HRMS (EI) calcd for $\mathrm{M}^{+\cdot}\left(\mathrm{C}_{8} \mathrm{H}_{13} \mathrm{O}_{2} \mathrm{~S}^{79} \mathrm{Br}\right)$ : 251.98196; found: 251.98109.

trimethyl[(2E)-3-(vinylsulfonyl)prop-2-en-1-yl]silane (7i)

Pale brown oil (66 \%). IR (film): $\nu$ 3053, 2961, 2926, 2855, 1740, 1614, 1412, 1317, 1294, 1261, 1098, 1028, 801, 724, 699, 640, $500 \mathrm{~cm}^{-1} .{ }^{1} \mathrm{H}$ NMR (400 MHz, $\left.\mathrm{CDCl}_{3}\right) \delta 0.08$ (s, 9H), 1.83 (dd, $J=9.1,1.3 \mathrm{~Hz}, 2 \mathrm{H}), 6.00(\mathrm{~d}, J=9.7 \mathrm{~Hz}, 1 \mathrm{H}), 6.05(\mathrm{dt}, J=14.8,1.3 \mathrm{~Hz}, 1 \mathrm{H}), 6.34(\mathrm{~d}, J=16.6$ $\mathrm{Hz}, 1 \mathrm{H}), 6.57(\mathrm{dd}, J=16.6,9.7 \mathrm{~Hz}, 1 \mathrm{H}), 6.99(\mathrm{dt}, J=14.8,9.1 \mathrm{~Hz}, 1 \mathrm{H}) \mathrm{ppm} .{ }^{13} \mathrm{C}$ NMR $(100$ $\left.\mathrm{MHz}, \mathrm{CDCl}_{3}\right) \delta-1.9,24.7,125.4,127.2,138.3,148.7$. MS (EI) $\mathrm{m} / z$ (rel intensity) 188 (3), 187 (17), 122 (12), 113 (5), 97 (32), 75 (11), 73 (100), 67 (15), 59 (8), 45 (7). HRMS (ESI) calcd for $[\mathrm{M}+\mathrm{Na}]^{+} \quad\left(\mathrm{C}_{8} \mathrm{H}_{16} \mathrm{O}_{2} \mathrm{SSiNa}\right): 227.0533$; found: 227.0521 .

\section{1,1'-[Sulfonyldi(1E)prop-1-ene-1,3-diyl]dibenzene (8b).}

Brown oil (9 \%). IR (KBr): $\nu$ 3063, 3044, 3028, 2923, 1727, 1634, 1599, 1495, 1453, 1437, 1307, $1273,1122,983,929,856,800,750,724,699,612,570,523,493,477 \mathrm{~cm}^{-1} .{ }^{1} \mathrm{H}$ NMR $(500 \mathrm{MHz}$, $\left.\mathrm{CDCl}_{3}\right) \delta 3.55(\mathrm{dd}, J=6.5,1.7 \mathrm{~Hz}, 4 \mathrm{H}), 6.15(\mathrm{dt}, J=15.1,1.7 \mathrm{~Hz}, 2 \mathrm{H}), 7.03(\mathrm{dt}, J=15.1,6.5$ $\mathrm{Hz}, 2 \mathrm{H}), 7.12-7.16(\mathrm{~m}, 4 \mathrm{H}), 7.24-7.28(\mathrm{~m}, 2 \mathrm{H}), 7.30-7.34(\mathrm{~m}, 4 \mathrm{H}) \mathrm{ppm} .{ }^{13} \mathrm{C} \mathrm{NMR}(125 \mathrm{MHz}$, $\left.\mathrm{CDCl}_{3}\right) \delta 37.7,127.1,128.8,128.9,130.5,136.2,146.4$. MS (EI) $\mathrm{m} / \mathrm{z}$ (rel intensity) $298\left(40, \mathrm{M}^{+\cdot}\right.$ ), 281 (5), 232 (8), 147 (4), 118 (10), 117 (100), 116 (25), 115 (38), 91 (16), 65 (4). HRMS (EI) calcd for $\mathrm{M}^{+\cdot}\left(\mathrm{C}_{18} \mathrm{H}_{18} \mathrm{O}_{2} \mathrm{~S}\right)$ : 298.10275; found: 298.10367 .

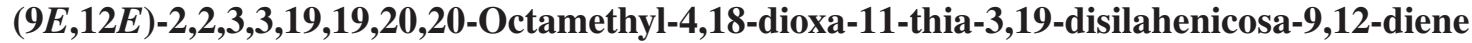 11,11-dioxide (8e)}

Yellow oil (13\%). IR (film): $\nu$ 2952, 2930, 2886, 2858, 1633, 1472, 1463, 1388, 1321, 1255, 1130, 1101, 1005, 976, 836, 775, 661, $607 \mathrm{~cm}^{-1} .{ }^{1} \mathrm{H}$ NMR $\left(500 \mathrm{MHz}, \mathrm{CDCl}_{3}\right) \delta 0.04(\mathrm{~s}, 12 \mathrm{H}), 0.88$ $(\mathrm{s}, 18 \mathrm{H}), 1.51-1.56(\mathrm{~m}, 8 \mathrm{H}), 2.23-2.31(\mathrm{~m}, 4 \mathrm{H}), 3.58-3.63(\mathrm{~m}, 4 \mathrm{H}), 6.22(\mathrm{dt}, J=15.1,1.4 \mathrm{~Hz}$, $2 \mathrm{H}), 6.88(\mathrm{dt}, J=15.1,6.8 \mathrm{~Hz}, 2 \mathrm{H}) \mathrm{ppm} .{ }^{13} \mathrm{C} \mathrm{NMR}\left(125 \mathrm{MHz}, \mathrm{CDCl}_{3}\right) \delta-5.3,18.3,24.1,25.9$, 31.3, 32.0, 62.5, 129.6, 147.6. MS (EI) $\mathrm{m} / \mathrm{z}$ (rel intensity) 476 (4), 435 (17), 434 (33), 433 (100), 221 (3), 149 (5), 115 (3), 81 (26), 75 (20), 73 (20), 57 (5), 55 (5), 41 (6). HRMS (ESI) calcd for $[\mathrm{M}+\mathrm{Na}]^{+}\left(\mathrm{C}_{8} \mathrm{H}_{16} \mathrm{O}_{2} \mathrm{SSiNa}\right)$ : 513.2861; found: 513.2883. Anal. calcd for $\mathrm{C}_{24} \mathrm{H}_{50} \mathrm{O}_{4} \mathrm{Si}_{2} \mathrm{~S}$ (490.90): C 58.72, H 10.27, S 6.53; found C 58.77, H 10.37, S 6.54.

\section{(10E,10'E)-11,11'-Sulfonylbisundec-10-en-1-ol (8f)}

Yellow solid (12 \%). IR (KBr): $\nu$ 3410, 3344, 2926, 2850, 1638, 1465, 1371, 1317, 1306, 1281, $1265,1120,1063,1003,972,843,810,728,627,535,492 \mathrm{~cm}^{-1} .{ }^{1} \mathrm{H}$ NMR $\left(500 \mathrm{MHz}, \mathrm{CDCl}_{3}\right) \delta$ $1.25-1.36(\mathrm{~m}, 22 \mathrm{H}), 1.43-1.50(\mathrm{~m}, 4 \mathrm{H}), 1.51-1.59(\mathrm{~m}, 4 \mathrm{H}), 2.24(\mathrm{qd}, J=6.8,1.5 \mathrm{~Hz}, 4 \mathrm{H}), 3.62$ $(\mathrm{t}, J=6.6 \mathrm{~Hz}, 4 \mathrm{H}), 6.20(\mathrm{dt}, J=15.1,1.5 \mathrm{~Hz}, 4 \mathrm{H}), 6.86(\mathrm{dt}, J=15.1,6.8 \mathrm{~Hz}, 4 \mathrm{H}) \mathrm{ppm} .{ }^{13} \mathrm{C}$ NMR $\left(125 \mathrm{MHz}, \mathrm{CDCl}_{3}\right) \delta 25.6,27.6,28.9,29.1,29.2,29.3,31.5,32.7,62.9,129.5,147.8$. HRMS (ESI) calcd for $[\mathrm{M}+\mathrm{Na}]^{+}\left(\mathrm{C}_{22} \mathrm{H}_{42} \mathrm{O}_{4} \mathrm{SNa}\right): 425.2696$; found: 425.2715 .

\section{(1E,1'E)-1,1'-Sulfonylbis(10-bromodec-1-ene) (8g)}

Pale brown oil (19 \%). IR (film): $\nu$ 2929, 2856, 1629, 1582, 1516, 1463, 1339, 1318, 1271, 1129, 968, 834, 756, 609, $558 \mathrm{~cm}^{-1} .{ }^{1} \mathrm{H}$ NMR $\left(500 \mathrm{MHz}, \mathrm{CDCl}_{3}\right) \delta 1.25-1.35(\mathrm{~m}, 12 \mathrm{H}), 1.38-1.51(\mathrm{~m}$, $8 \mathrm{H}), 1.81-1.88(\mathrm{~m}, 4 \mathrm{H}), 2.24(\mathrm{qd}, J=6.8,1.4 \mathrm{~Hz}, 4 \mathrm{H}), 3.40(\mathrm{t}, J=6.8 \mathrm{~Hz}, 4 \mathrm{H}), 6.21(\mathrm{dt}, J=$ $15.1,1.4 \mathrm{~Hz}, 2 \mathrm{H}), 6.87$ (dt, $J=15.1,6.8 \mathrm{~Hz}, 2 \mathrm{H}) \mathrm{ppm} .{ }^{13} \mathrm{C} \mathrm{NMR}\left(125 \mathrm{MHz}, \mathrm{CDCl}_{3}\right) \delta 27.6,28.0$, 28.5, 28.9, 29.1, 31.5, 32.7, 33.9, 129.6, 147.7. MS (EI) $\mathrm{m} / \mathrm{z}$ (rel intensity) $500\left(2, \mathbf{M}^{+\cdot}\right.$ ), 483 (2), 481 (1), 324 (7), 285 (26), 283 (25), 137 (100), 136 (27), 109 (18), 95 (80), 82 (21), 81 (94), 69 (37), 67 (53), 55 (50), 41 (35). HRMS (EI) calcd for $\mathrm{M}^{+\cdot}\left(\mathrm{C}_{20} \mathrm{H}_{36} \mathrm{O}_{2} \mathrm{~S}^{79} \mathrm{Br}^{81} \mathrm{Br}\right)$ : 500.07823; found: 500.08063.

[Sulfonyldi(1E)prop-1-ene-1,3-diyl]bis(trimethylsilane) (8i)

Colourless oil (31\%). ${ }^{1} \mathrm{H}$ NMR $\left(400 \mathrm{MHz}, \mathrm{CDCl}_{3}\right) \delta 0.07(\mathrm{~s}, 18 \mathrm{H}), 1.77$ (dd, $J=9.0,1.3 \mathrm{~Hz}$, $4 \mathrm{H}), 6.02(\mathrm{dt}, J=14.9,1.3 \mathrm{~Hz}, 2 \mathrm{H}), 6.89(\mathrm{dt}, J=14.9,9.0 \mathrm{~Hz}, 2 \mathrm{H}) \mathrm{ppm} .{ }^{13} \mathrm{C} \mathrm{NMR}(100 \mathrm{MHz}$, $\left.\mathrm{CDCl}_{3}\right) \delta-1.9,24.2,127.3,145.8$. MS (EI) $\mathrm{m} / z$ (rel intensity) $290\left(1, \mathrm{M}^{+\cdot}\right), 210$ (7), 161 (4), 147 (8), 133 (3), 111 (20), 79 (6), 75 (6), 73 (100), 45 (11). HRMS (EI) calcd for $\mathrm{M}^{+\cdot}\left(\mathrm{C}_{12} \mathrm{H}_{26} \mathrm{O}_{2} \mathrm{Si}_{2} \mathrm{~S}\right.$ ): 290.11997; found: 290.11921. 


\subsection{Representative procedure for $\mathrm{CM}$ of sulfones 7}

To a mixture of alkene $(8.0 \mathrm{mmol})$ and sulfone $7(2.0 \mathrm{mmol})$ in $\mathrm{CH}_{2} \mathrm{Cl}_{2}(100 \mathrm{~mL}, c=0.02 \mathrm{M})$ was added a Ru-catalyst $\mathbf{1}$ or $\mathbf{4}$ as a solid (20-8 mol\%). The resulted mixture was stirred at reflux for 16-44 $\mathrm{h}$ under Ar. The solvent was removed under reduced pressure. The crude product was purified by flash chromatography using $c$-hexan/ethyl acetate as eluent (Merck silica gel 60, 230-400 mesh).

\section{(10E)-11-[(1E)-10-bromodec-1-en-1-yl]sulfonylundec-10-en-1-ol (10fg)}

Colourless solid (74 \%). IR (KBr): $\nu$ 3421, 3057, 2931, 2852, 1638, 1466, 1316, 1279, 1118, 1061, $963,851,832,811,729,649,625,533,486 \mathrm{~cm}^{-1} .{ }^{1} \mathrm{H}$ NMR $\left(500 \mathrm{MHz}, \mathrm{CDCl}_{3}\right) \delta 1.26-1.36(\mathrm{~m}$, $16 \mathrm{H}), 1.38-1.50(\mathrm{~m}, 6 \mathrm{H}), 1.52-1.59(\mathrm{~m}, 2 \mathrm{H}), 1.81-1.88(\mathrm{~m}, 2 \mathrm{H}), 2.24(\mathrm{q}, J=7.3 \mathrm{~Hz}, 4 \mathrm{H}), 3.40$ $(\mathrm{t}, J=6.8 \mathrm{~Hz}, 2 \mathrm{H}), 3.60-3.66(\mathrm{~m}, 2 \mathrm{H}), 6.20(\mathrm{~d}, J=15.1 \mathrm{~Hz}, 2 \mathrm{H}), 6.83-6.90(\mathrm{~m}, 2 \mathrm{H}) \mathrm{ppm} .{ }^{13} \mathrm{C}$ NMR $\left(125 \mathrm{MHz}, \mathrm{CDCl}_{3}\right) \delta 25.7,27.6,28.0,28.5,28.9,29.0,29.1,29.2,29.3,29.4,31.5,32.7$, 33.9, 63.0, 129.5, 129.6, 147.6, 147.8. MS (EI) $\mathrm{m} / \mathrm{z}$ (rel intensity) 422 (15), 420 (14), 405 (9), 403 (8), 337 (11), 335 (10), 307 (10), 305 (10), 285 (59), 283 (57), 217 (17), 215 (12), 168 (12), 151 (68), 150 (60), 137 (99), 135 (32), 121 (43), 109 (56), 107 (24), 95 (100), 81 (69), 67 (41), 55 (43), 41 (39). HRMS (ESI) calcd for [M+Na] ${ }^{+}\left(\mathrm{C}_{21} \mathrm{H}_{39} \mathrm{O}_{3} \mathrm{~S}^{79} \mathrm{BrNa}\right)$ : 473.1695 ; found: 473.1699. Anal. calcd for $\mathrm{C}_{21} \mathrm{H}_{39} \mathrm{O}_{3} \mathrm{SBr}$ (451.51): C 55.86, $\mathrm{H}$ 8.71, S 7.10; found $\mathrm{C} 56.08, \mathrm{H}$ 8.91, S 7.05.

\section{(1E)-Nonadec-1-en-1-yl (1E)-3-phenylprop-1-en-1-yl sulfone (10db)}

Yellow oil (54 \%). IR (KBr): $\nu$ 2957, 2921, 2848, 1640, 1462, 1321, 1278, 1119, 964, 850, 826, $803,744,724,698,624,578,486 \mathrm{~cm}^{-1} .{ }^{1} \mathrm{H}$ NMR $\left(500 \mathrm{MHz}, \mathrm{CDCl}_{3}\right) \delta 0.91(\mathrm{t}, J=7.1 \mathrm{~Hz}, 3 \mathrm{H})$, $1.26-1.34(\mathrm{~m}, 28 \mathrm{H}), 1.42-1.54(\mathrm{~m}, 2 \mathrm{H}), 2.23-2.30(\mathrm{~m}, 2 \mathrm{H}), 3.59(\mathrm{dd}, J=6.4,1.5 \mathrm{~Hz}, 2 \mathrm{H}), 6.18$ $(\mathrm{dt}, J=15.1,1.7 \mathrm{~Hz}, 1 \mathrm{H}), 6.23(\mathrm{dt}, J=15.1,1.5 \mathrm{~Hz}, 1 \mathrm{H}), 6.86-6.94(\mathrm{~m}, 1 \mathrm{H}), 7.07(\mathrm{dt}, J=15.1$, $6.4 \mathrm{~Hz}, 1 \mathrm{H}), 7.17-7.21(\mathrm{~m}, 2 \mathrm{H}), 7.27-7.43(\mathrm{~m}, 3 \mathrm{H}) \mathrm{ppm} .{ }^{13} \mathrm{C} \mathrm{NMR}\left(125 \mathrm{MHz}, \mathrm{CDCl}_{3}\right) \delta 14.1$, 22.7, 27.6, 29.0, 29.3, 29.5, 29.6, 29.7, 31.6, 31.9, 37.7, 127.1, 128.8, 128.9, 129.2, 130.8, 136.3, 145.9, 148.3. MS (EI) $\mathrm{m} / \mathrm{z}$ (rel intensity) $446\left(1, \mathrm{M}^{+\cdot}\right), 355$ (2), 118 (10), 117 (100), 115 (7), 91 (3), 43 (2). HRMS (EI) calcd for $\mathrm{M}^{+\cdot}\left(\mathrm{C}_{28} \mathrm{H}_{46} \mathrm{O}_{2} \mathrm{~S}\right)$ : 446.32185; found: 446.32201.

Tert-butyl((5E)-6-[(1E)-hept-1-en-1-ylsulfonyl]hex-5-en-1-yloxy)dimethylsilane (10ec) Yellow oil (85 \%). IR (KBr): $\nu$ 2955, 2930, 2858, 1634, 1471, 1463, 1320, 1256, 1131, 1102, 977, 836, 776, 608, $548 \mathrm{~cm}^{-1} .{ }^{1} \mathrm{H}$ NMR $\left(500 \mathrm{MHz}, \mathrm{CDCl}_{3}\right) \delta 0.04(\mathrm{~s}, 6 \mathrm{H}), 0.86-0.91(\mathrm{~m}, 12 \mathrm{H}), 1.26-$ $1.34(\mathrm{~m}, 4 \mathrm{H}), 1.44-1.51(\mathrm{~m}, 2 \mathrm{H}), 1.51-1.57(\mathrm{~m}, 4 \mathrm{H}), 2.20-2.30(\mathrm{~m}, 4 \mathrm{H}), 3.58-3.63(\mathrm{~m}, 2 \mathrm{H}), 6.20$ $(\mathrm{dt}, J=15.1,1.5 \mathrm{~Hz}, 1 \mathrm{H}), 6.22(\mathrm{dt}, J=15.1,1.5 \mathrm{~Hz}, 1 \mathrm{H}), 6.87(\mathrm{dt}, J=15.1,6.7 \mathrm{~Hz}, 1 \mathrm{H}) \mathrm{ppm}$. ${ }^{13} \mathrm{C}$ NMR $\left(125 \mathrm{MHz}, \mathrm{CDCl}_{3}\right) \delta-5.3,13.9,18.3,22.3,24.1,25.9,27.3,31.2,31.3,31.5,32.1,62.6$, 129.5, 129.7, 147.5, 147.9. MS (EI) $\mathrm{m} / z$ (rel intensity) 359 (3), 319 (11), 318 (24), 317 (100), 155 (17), 95 (3), 81 (5), 75 (15), 73 (8), 41 (5). HRMS (ESI) calcd for $[\mathrm{M}+\mathrm{Na}]^{+} \quad\left(\mathrm{C}_{19} \mathrm{H}_{38} \mathrm{O}_{3} \mathrm{SSiNa}\right)$ : 397.2203; found: 397.2224 .

(1E)-3-(4-Bromophenyl)prop-1-en-1-yl (1E)-3-phenylprop-1-en-1-yl sulfone (10bj)

Yellow solid (74 \%). IR (film): $\nu$ 3047, 3028, 2904, 1630, 1488, 1422, 1316, 1282, 1127, 1072, 1012, 976, 858, 818, 794, 751, 700, 629, 603, $513 \mathrm{~cm}^{-1} .{ }^{1} \mathrm{H}$ NMR $\left(500 \mathrm{MHz}, \mathrm{CDCl}_{3}\right) \delta 3.50$ $(\mathrm{dd}, J=6.4,1.5 \mathrm{~Hz}, 2 \mathrm{H}), 3.56(\mathrm{dd}, J=6.4,1.5 \mathrm{~Hz}, 2 \mathrm{H}), 6.10-6.16(\mathrm{~m}, 2 \mathrm{H}), 6.95-7.08(\mathrm{~m}, 4 \mathrm{H})$, $7.14(\mathrm{~d}, J=7.0 \mathrm{~Hz}, 2 \mathrm{H}), 7.24-7.35(\mathrm{~m}, 3 \mathrm{H}), 7.45(\mathrm{~d}, J=8.4 \mathrm{~Hz}, 2 \mathrm{H}) \mathrm{ppm} .{ }^{13} \mathrm{C}$ NMR $(125 \mathrm{MHz}$, $\left.\mathrm{CDCl}_{3}\right) \delta 37.0,37.7,121.1,127.1,128.8,128.9,130.3,130.5,131.0,132.0,135.2,136.2,145.5$, 146.7. MS (EI) $\mathrm{m} / \mathrm{z}$ (rel intensity) $378\left(100, \mathrm{M}^{+\cdot}\right), 376$ (95), 312 (16), 310 (17), 197 (65), 195 (70), 171 (21), 169 (21), 117 (81), 116 (89), 115 (66) 91 (21), 89 (10), 65 (8). HRMS (EI) calcd for $\mathrm{M}^{+\cdot}\left(\mathrm{C}_{18} \mathrm{H}_{17} \mathrm{O}_{2} \mathrm{~S}^{79} \mathrm{Br}\right)$ : 376.01326; found: 376.01312. Anal. calcd for $\mathrm{C}_{18} \mathrm{H}_{17} \mathrm{O}_{2} \mathrm{SBr}$ (377.30): C 57.30, H 4.54, S 8.50; found C 57.19, H 4.60, S 8.40.

\subsection{Preparation of thiomorpholine 1,1-dioxides}

Neat benzylamine or hydrazine hydrate $(0.1 \mathrm{mmol})$ was added to the solution of sulfone $\mathbf{7 , 8}$ or $\mathbf{1 0}$ $(0.1 \mathrm{mmol})$ in a mixture of propan-2-ol $(10 \mathrm{~mL})$ and $\mathrm{H}_{2} \mathrm{O}(5 \mathrm{~mL})$ and the reaction was refluxed for $24 \mathrm{~h}$. After cooling the reaction mixture to room temperature the solvents were removed under reduced pressure. The crude product was purified by flash chromatography using $c$-hexan/ethyl 
acetate as eluent (Merck silica gel 60, 230-400 mesh). ${ }^{1}$

\section{4-Benzyl-3-heptadecylthiomorpholine 1,1-dioxide (11a)}

Yellow solid (80 \%). IR (KBr): $\nu$ 2955, 2919, 2850, 1474, 1467, 1340, 1295, 1129, 985, 741, 704, $681,519 \mathrm{~cm}^{-1} .{ }^{1} \mathrm{H}$ NMR $\left(400 \mathrm{MHz}, \mathrm{CDCl}_{3}\right) \delta 0.88(\mathrm{t}, J=6.8 \mathrm{~Hz}, 3 \mathrm{H}), 1.20-1.34(\mathrm{~m}, 28 \mathrm{H})$, 1.36-1.46 (m, 2H), 1.60-1.82 (m, 2H), 2.78-2.86 (m, 1H), 2.92-3.09 (m, 4H), 3.20-3.26 (m, 1H), $3.27-3.35(\mathrm{~m}, 1 \mathrm{H}), 3.66(\mathrm{~d}, J=13.5 \mathrm{~Hz}, 1 \mathrm{H}), 3.72(\mathrm{~d}, J=13.5 \mathrm{~Hz}, 1 \mathrm{H}), 7.27-7.37(\mathrm{~m}, 5 \mathrm{H}) \mathrm{ppm}$. ${ }^{13} \mathrm{C}$ NMR $\left(125 \mathrm{MHz}, \mathrm{CDCl}_{3}\right) \delta 14.1,22.7,25.6,29.3,29.4,29.5,29.6,29.7,31.4,31.9,46.5,47.4$, 51.4, 52.4, 59.2, 127.6, 128.4, 128.6, 137.9. MS (EI) $\mathrm{m} / z$ (rel intensity) 252 (2), 226 (6), 225 (13), 224 (100), 133 (4), 92 (7), 91 (87), 69 (3), 57 (6) 55 (6), 43 (13), 41 (9). HRMS (EI) calcd for $\mathrm{M}^{+\cdot}\left(\mathrm{C}_{28} \mathrm{H}_{49} \mathrm{O}_{2} \mathrm{NS}\right)$ : 463.34840 ; found: 463.34754.

\section{4-Benzyl-3-(4-[tert-butyl(dimethyl)silyl]oxybutyl)thiomorpholine 1,1-dioxide (11b)}

Yellow solid (83 \%). IR (film): $\nu$ 3029, 2949, 2929, 2858, 1471, 1386, 1361, 1286, 1254, 1125, $1101,1003,835,774,731,699,679,511,440,432 \mathrm{~cm}^{-1} .{ }^{1} \mathrm{H}$ NMR $\left(500 \mathrm{MHz}, \mathrm{CDCl}_{3}\right) \delta 0.04(\mathrm{~s}$, $6 \mathrm{H}), 0.88(\mathrm{~s}, 9 \mathrm{H}), 1.45-1.60(\mathrm{~m}, 4 \mathrm{H}), 1.64-1.74(\mathrm{~m}, 1 \mathrm{H}), 1.75-1.90(\mathrm{~m}, 1 \mathrm{H}), 2.77-2.85(\mathrm{~m}, 1 \mathrm{H})$, 2.93-3.07 (m, 4H), 3.20-3.28 (m, 1H), 3.28-3.35 (m, 1H), $3.62(\mathrm{t}, J=6.1 \mathrm{~Hz}, 2 \mathrm{H}), 3.67(\mathrm{~d}, J=$ $13.5 \mathrm{~Hz}, 1 \mathrm{H}), 3.72(\mathrm{~d}, J=13.5 \mathrm{~Hz}, 1 \mathrm{H}), 7.25-7.35(\mathrm{~m}, 5 \mathrm{H}) \mathrm{ppm} .{ }^{13} \mathrm{C} \mathrm{NMR}\left(125 \mathrm{MHz}, \mathrm{CDCl}_{3}\right) \delta$ $-5.3,18.3,22.1,25.9,31.2,32.5,46.5,47.4,51.5,52.4,59.2,62.8,127.6,128.4,128.6,137.9$. MS (EI) $\mathrm{m} / \mathrm{z}$ (rel intensity) $411\left(1, \mathbf{M}^{+\cdot}\right), 396$ (3), 355 (14), 354 (53), 339 (5), 224 (53), 174 (5), 133 (5), 91 (100), 73 (5). Anal. calcd for $\mathrm{C}_{21} \mathrm{H}_{37} \mathrm{NO}_{3} \mathrm{SSi}$ (411.68): C 61.27, H 9.06, N 3.40, S 7.79; found C 61.48, H 8.98, N 3.31, S 7.58.

\section{4-Benzyl-3-(4-[tert-butyl(dimethyl)silyl]oxybutyl)-5-pentylthiomorpholine 1,1-dioxide (11c)} Colourless oil (71 \%). IR (film): $\nu$ 3031, 2954, 2930, 2858, 2949, 2929, 2858, 1603, 1494, 1462, 1360, 1300, 1255, 1133, 1099, 1027, 836, 776, 726, 697, $523 \mathrm{~cm}^{-1} \cdot{ }^{1} \mathrm{H}$ NMR $\left(500 \mathrm{MHz}, \mathrm{CDCl}_{3}\right)$ $\delta \quad 0.04(\mathrm{~s}, 6 \mathrm{H}), 0.82(\mathrm{t}, J=6.8 \mathrm{~Hz}, 3 \mathrm{H}), 0.87(\mathrm{~s}, 9 \mathrm{H}), 1.15-1.38(\mathrm{~m}, 8 \mathrm{H}), 1.45-1.65(\mathrm{~m}, 4 \mathrm{H})$, 1.70-1.77 (m, 1H), 1.85-1.98 (m, 1H), 2.78-2.86 (m, 2H), 2.92-3.01 (m, 2H), 3.37-3.44 (m, 2H), $3.59(\mathrm{t}, J=6.2 \mathrm{~Hz}, 2 \mathrm{H}), 3.65(\mathrm{~s}, 2 \mathrm{H}), 7.19-7.23(\mathrm{~m}, 1 \mathrm{H}), 7.27-7.33(\mathrm{~m}, 2 \mathrm{H}), 7.34-7.38(\mathrm{~m}, 2 \mathrm{H})$ ppm. ${ }^{13} \mathrm{C}$ NMR $\left(125 \mathrm{MHz}, \mathrm{CDCl}_{3}\right) \delta-5.3,13.9,18.3,22.4,23.0,25.9,26.2,31.4,32.3,33.7,33.9$, 44.8, 50.3, 50.4, 61.7, 62.7, 126.7, 127.2, 128.2, 141.0. MS (EI) $\mathrm{m} / \mathrm{z}$ (rel intensity) 466 (2), 424 (19), 411 (5), 410 (18), 295 (11), 294 (62), 92 (7), 91 (100), 75 (4). Anal. calcd for $\mathrm{C}_{26} \mathrm{H}_{47} \mathrm{NO}_{3} \mathrm{SSi}$ (481.82): C 64.81, H 9.83, N 2.91, S 6.65; found C 64.82, H 9.97, N 2.95, S 6.54.

\section{3-Heptadecylthiomorpholin-4-amine 1,1-dioxide (11d)}

Colourless solid (66 \%). IR (KBr): $\nu$ 2849, 1472, 1291, 1127, 868, 717, 684, 520, $449 \mathrm{~cm}^{-1} \cdot{ }^{1} \mathrm{H}$ NMR $\left(400 \mathrm{MHz}, \mathrm{CDCl}_{3}\right) \delta 0.88(\mathrm{t}, J=6.6 \mathrm{~Hz}, 3 \mathrm{H}), 1.18-1.40(\mathrm{~m}, 30 \mathrm{H}), 1.40-1.52(\mathrm{~m}, 1 \mathrm{H})$, $1.70-1.80(\mathrm{~m}, 1 \mathrm{H}), 2.80-2.90(\mathrm{~m}, 3 \mathrm{H}), 2.96-3.10(\mathrm{~m}, 3 \mathrm{H}), 3.27-3.34(\mathrm{~m}, 2 \mathrm{H}), 3.44-3.52(\mathrm{~m}, 1 \mathrm{H})$ ppm. ${ }^{13} \mathrm{C}$ NMR $\left(100 \mathrm{MHz}, \mathrm{CDCl}_{3}\right) \delta 14.1,22.7,25.1,29.5,29.6,29.7,47.6,51.8,55.3,62.4 . \mathrm{MS}$ (EI) $\mathrm{m} / z$ (rel intensity) $388\left(19, \mathbf{M}^{+\cdot}\right.$ ), 177 (2), 151 (5), 150 (6), 149 (100), 134 (13), 83 (3), 69 (5) 58 (6), 57 (9), 55 (9) 43 (16), 41 (13). Anal. calcd for $\mathrm{C}_{21} \mathrm{H}_{44} \mathrm{~N}_{2} \mathrm{O}_{2} \mathrm{~S}$ (388.6610): C 64.90, $\mathrm{H}$ 11.41, N 7.21, S 8.25; found C 64.69, H 11.65, N 7.14, S 8.17.

\section{3,5-Dibenzylthiomorpholin-4-amine 1,1-dioxide (11e)}

Colourless solid (48 \%). IR (KBr): $\nu$ 3477, 3346, 3060, 3026, 2968, 2952, 2922, 2853, 1681, 1603 , $1495,1454,1310,1278,1239,1140,1119,1012,994,972,873,745,698,596,512,464,433$ $\mathrm{cm}^{-1} .{ }^{1} \mathrm{H}$ NMR $\left(400 \mathrm{MHz}, \mathrm{CDCl}_{3}\right) \delta$ 2.64-2.76 (m, 4H), $2.82(\mathrm{~s}, 2 \mathrm{H}), 2.94 ? ? ? 3.60(\mathrm{~m}, 2 \mathrm{H}), 3.17$ $(\mathrm{dd}, J=13.9,6.7 \mathrm{~Hz}, 2 \mathrm{H}), 3.52-3.62(\mathrm{~m}, 2 \mathrm{H}), 7.10-7.16(\mathrm{~m}, 4 \mathrm{H}), 7.20-7.30(\mathrm{~m}, 6 \mathrm{H}) \mathrm{ppm} .{ }^{13} \mathrm{C}$ NMR (100 MHz, $\left.\mathrm{CDCl}_{3}\right) \delta 39.2,48.0,63.4,126.8,128.7,129.0,137.1 . \mathrm{MS}$ (EI) $\mathrm{m} / \mathrm{z}$ (rel intensity) 330 (4, M+·), 241 (6), 240 (14), 239 (100), 183 (10), 158 (3), 119 (5), 117 (36) 115 (6), 91 (21), 65 (4), 57 (31). HRMS (EI) calcd for $\mathrm{M}^{+\cdot}\left(\mathrm{C}_{18} \mathrm{H}_{22} \mathrm{O}_{2} \mathrm{~N}_{2} \mathrm{~S}\right)$ : 330.14020; found: 330.13973.

\section{9-[4-Amino-5-(8-bromooctyl)-1,1-dioxidothiomorpholin-3-yl]nonan-1-ol (11f)}

Yellow solid (17 \%). IR (KBr): $\nu$ 3369, 2929, 2850, 1740, 1468, 1333, 1291, 1151, 1132, 1059, 1011, 906, 890, 721, 649, 543, $452 \mathrm{~cm}^{-1} .{ }^{1} \mathrm{H}$ NMR (400 MHz, $\left.\mathrm{CDCl}_{3}\right) \delta 1.22-1.48$ (m, 24H),

\footnotetext{
${ }^{1}$ Teyssot, M.-L.; Fayolle, M.; Philouze, C.; Dupuy, C. Eur. J. Org. Chem. 2003, 54-62. 
$1.50-1.60(\mathrm{~m}, 2 \mathrm{H}), 1.68-1.80(\mathrm{~m}, 2 \mathrm{H}), 1.80-1.90(\mathrm{~m}, 2 \mathrm{H}), 2.64-2.72(\mathrm{~m}, 2 \mathrm{H}), 2.92-3.20(\mathrm{~m}, 2 \mathrm{H})$, $3.22-3.32(\mathrm{~m}, 2 \mathrm{H}), 3.40(\mathrm{t}, J=6.9 \mathrm{~Hz}, 2 \mathrm{H}), 3.64(\mathrm{t}, J=6.6 \mathrm{~Hz}, 2 \mathrm{H}) \mathrm{ppm} .{ }^{13} \mathrm{C} \mathrm{NMR}(100 \mathrm{MHz}$, $\left.\mathrm{CDCl}_{3}\right) \delta 25.8,25.9,26.0,28.2,28.8,29.3,29.4,29.5,29.6,29.7,32.9,34.2,49.0,61.6,63.0 . \mathrm{MS}$ (EI) $\mathrm{m} / \mathrm{z}$ (rel intensity) 484 (36, $\mathbf{M}^{+\cdot}$ ), 241 (6), 240 (14), 239 (100), 183 (10), 158 (3), 119 (5), 117 (36) 115 (6), 91 (21), 65 (4) 57 (31). HRMS (EI) calcd for $\mathrm{M}^{+\cdot}\left(\mathrm{C}_{21} \mathrm{H}_{43} \mathrm{O}_{3} \mathrm{~N}_{2} \mathrm{~S}^{79} \mathrm{Br}\right)$ : 482.21778; found: 482.21810 .

\subsection{Isomerisation of $8 \mathrm{~b}$}

1,1'-[Sulfonyldi(1E)prop-1-ene-3,1-diyl]dibenzene (8b'): sulfone $8 \mathbf{b}(0.1 \mathrm{mmol})$ and tetrabutylammonium hydroxide (40\% water solution, $0.1 \mathrm{mmol}$ ) were added to the solution of propan-2-ol $(10 \mathrm{~mL})$ and $\mathrm{H}_{2} \mathrm{O}(5 \mathrm{~mL})$ and reaction was refluxed for $24 \mathrm{~h}$. After cooling down the reaction mixture white crystals precipitated and were filtered off on Buchner funnel to give $17.3 \mathrm{mg}$ (55 $\%$ ) of the product as a colourless solid (55 \%). IR (KBr): $\nu$ 3050, 3030, 2976, 2926, 1596, 1576, 1497, 1449, 1414, 1320, 1290, 1277, 1147, 1115, 996, 978, 912, 759, 732, 714, 691, 596, 586, $484 \mathrm{~cm}^{-1} .{ }^{1} \mathrm{H}$ NMR $\left(400 \mathrm{MHz}, \mathrm{CDCl}_{3}\right) \delta 3.90(\mathrm{~d}, J=7.5 \mathrm{~Hz}, 4 \mathrm{H}), 6.27(\mathrm{dt}, J=15.8,7.5 \mathrm{~Hz}$, $2 \mathrm{H}), 6.68(\mathrm{~d}, J=15.8 \mathrm{~Hz}, 2 \mathrm{H}), 7.30-7.38(\mathrm{~m}, 6 \mathrm{H}), 7.39-7.43(\mathrm{~m}, 4 \mathrm{H}) \mathrm{ppm} .{ }^{13} \mathrm{C}$ NMR $(100 \mathrm{MHz}$, $\left.\mathrm{CDCl}_{3}\right) \delta 56.1,115.2,126.7,128.8,135.4,139.2$. MS (EI) $\mathrm{m} / \mathrm{z}$ (rel intensity) $298\left(1, \mathrm{M}^{+\cdot}\right), 234$ (1), 232 (1), 118 (10), 117 (100), 116 (7), 115 (23), 91 (10), 65 (2), 39 (3). HRMS (EI) calcd for $\mathrm{M}^{+\cdot}\left(\mathrm{C}_{18} \mathrm{H}_{18} \mathrm{O}_{2} \mathrm{~S}\right): 298.10275$; found: 298.10223 .

\section{Copies of ${ }^{1} \mathrm{H}$ and ${ }^{13} \mathrm{C}$-NMR spectra}

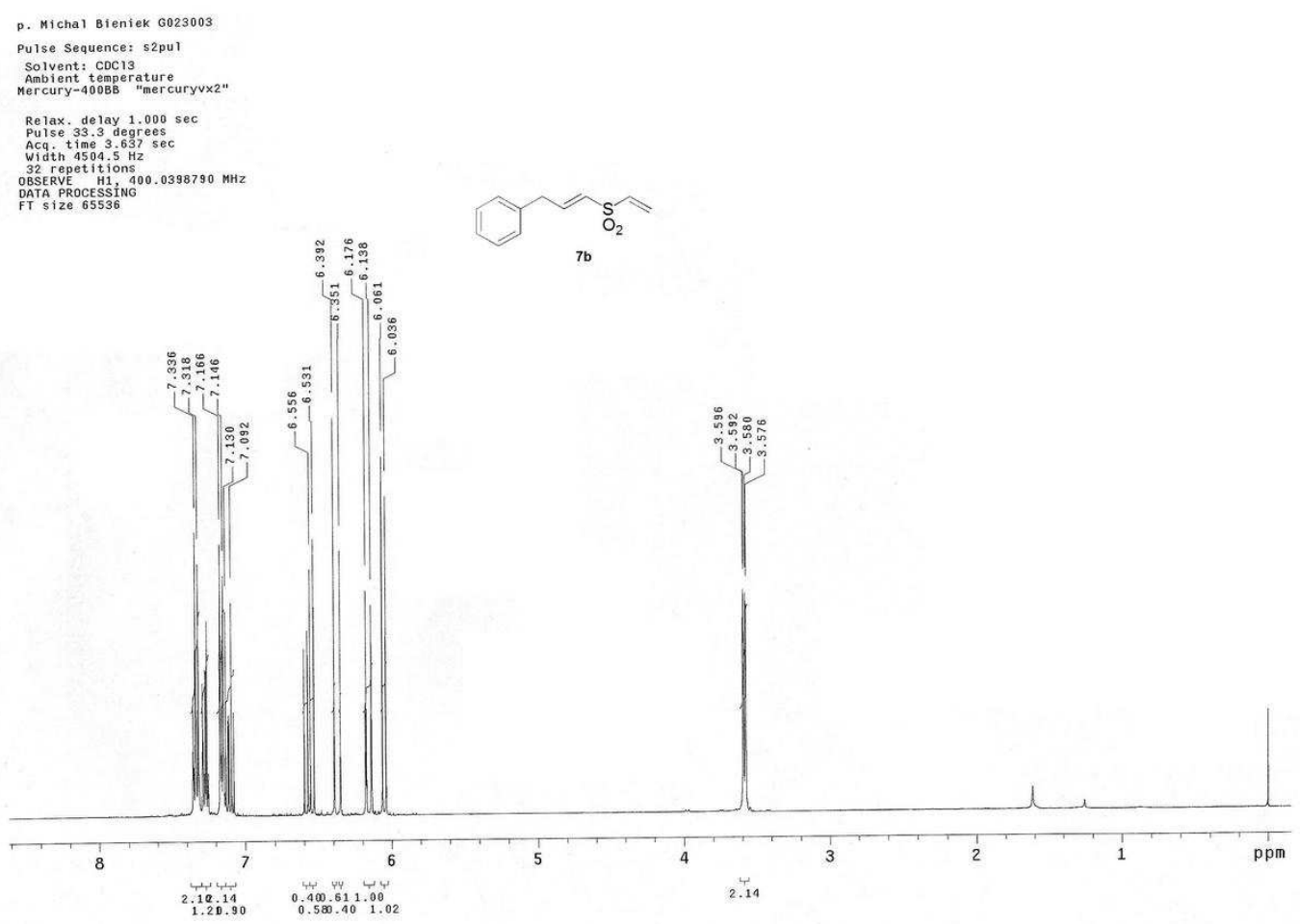



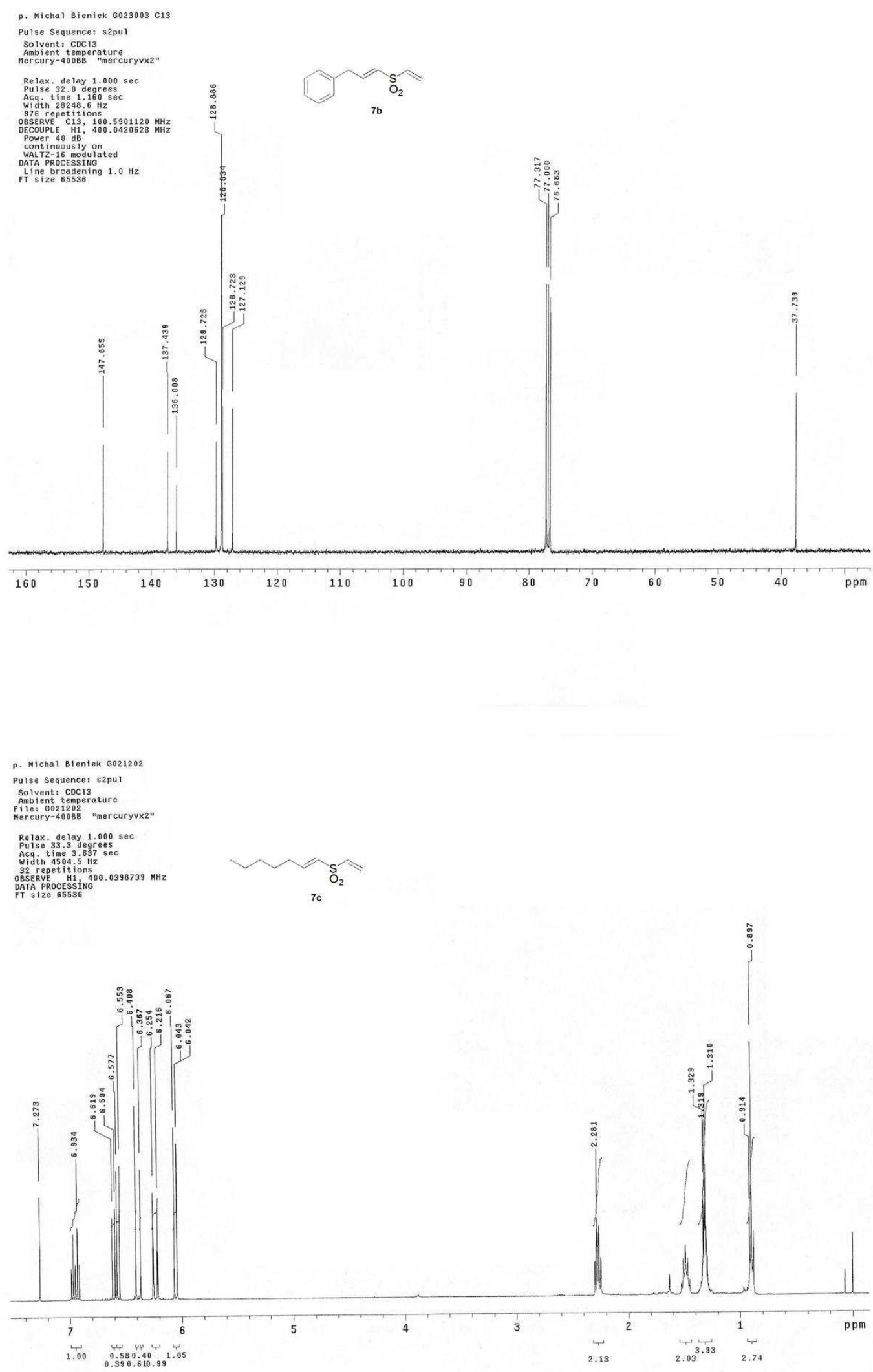

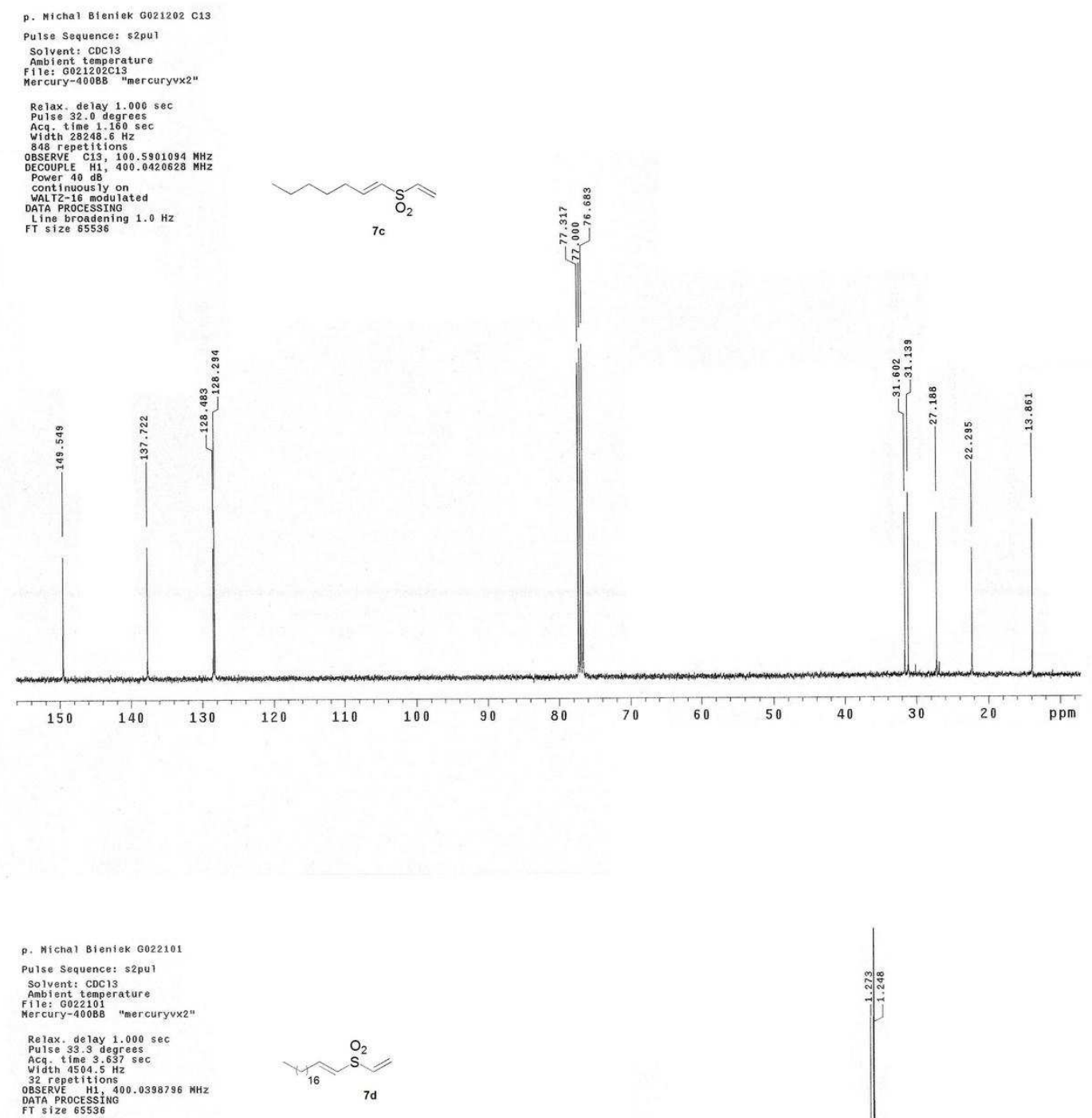

OATA PROCESSTNO
FT Size 65536

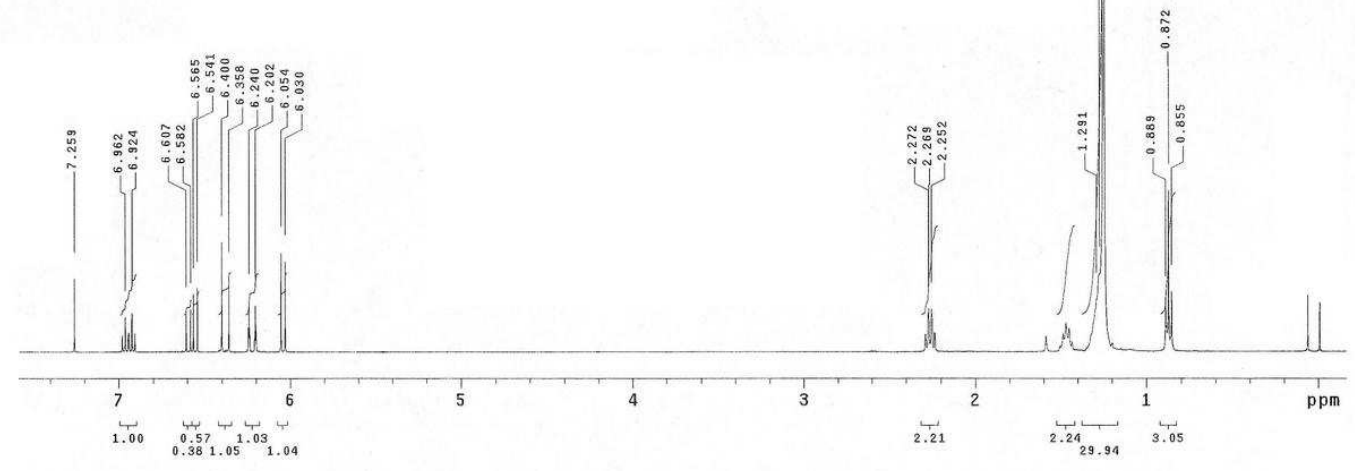



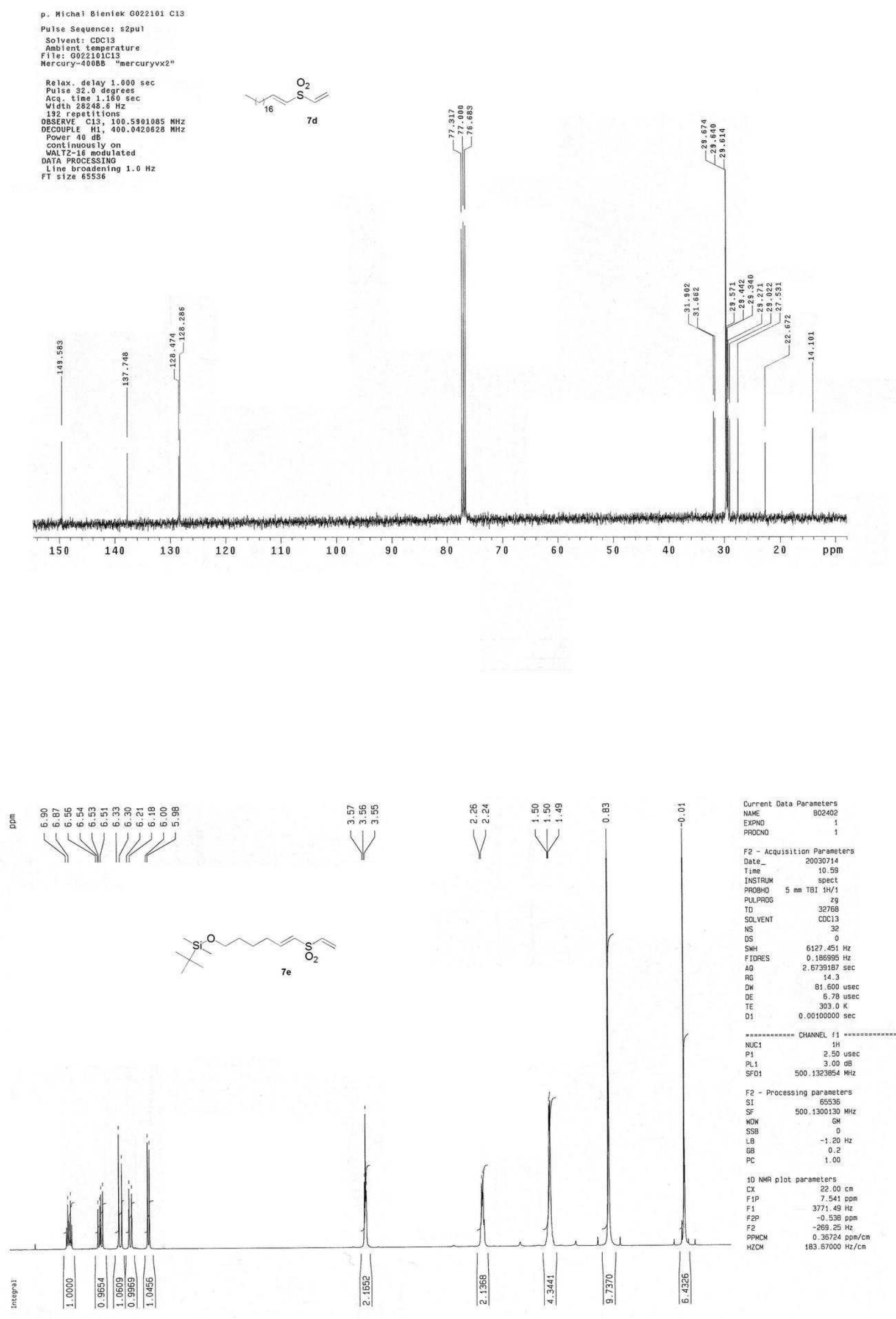

$\mathrm{Si}_{{ }_{7 e}} \mathrm{O}_{\mathrm{O}_{2}}^{\mathrm{O}}$ 

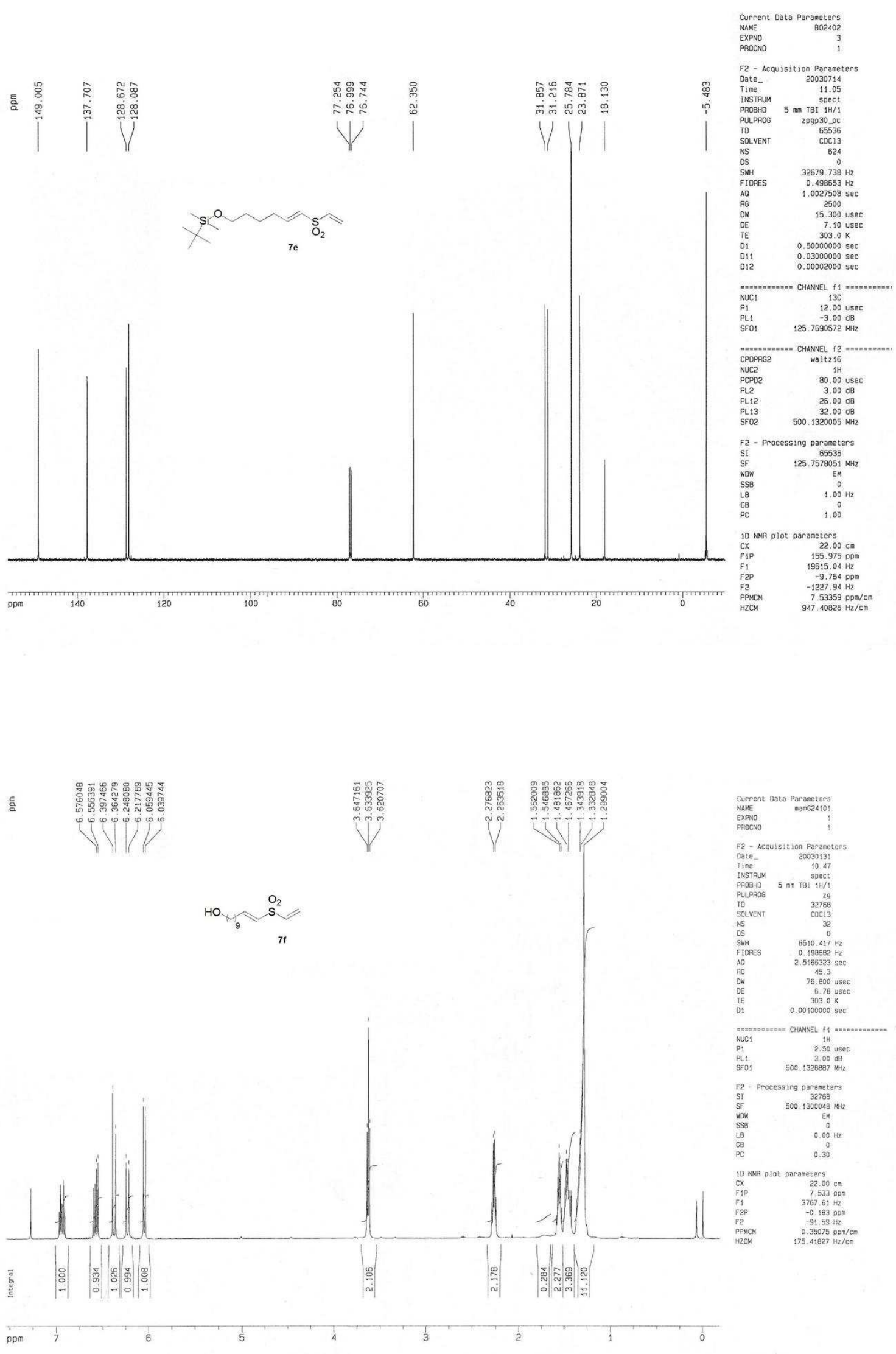

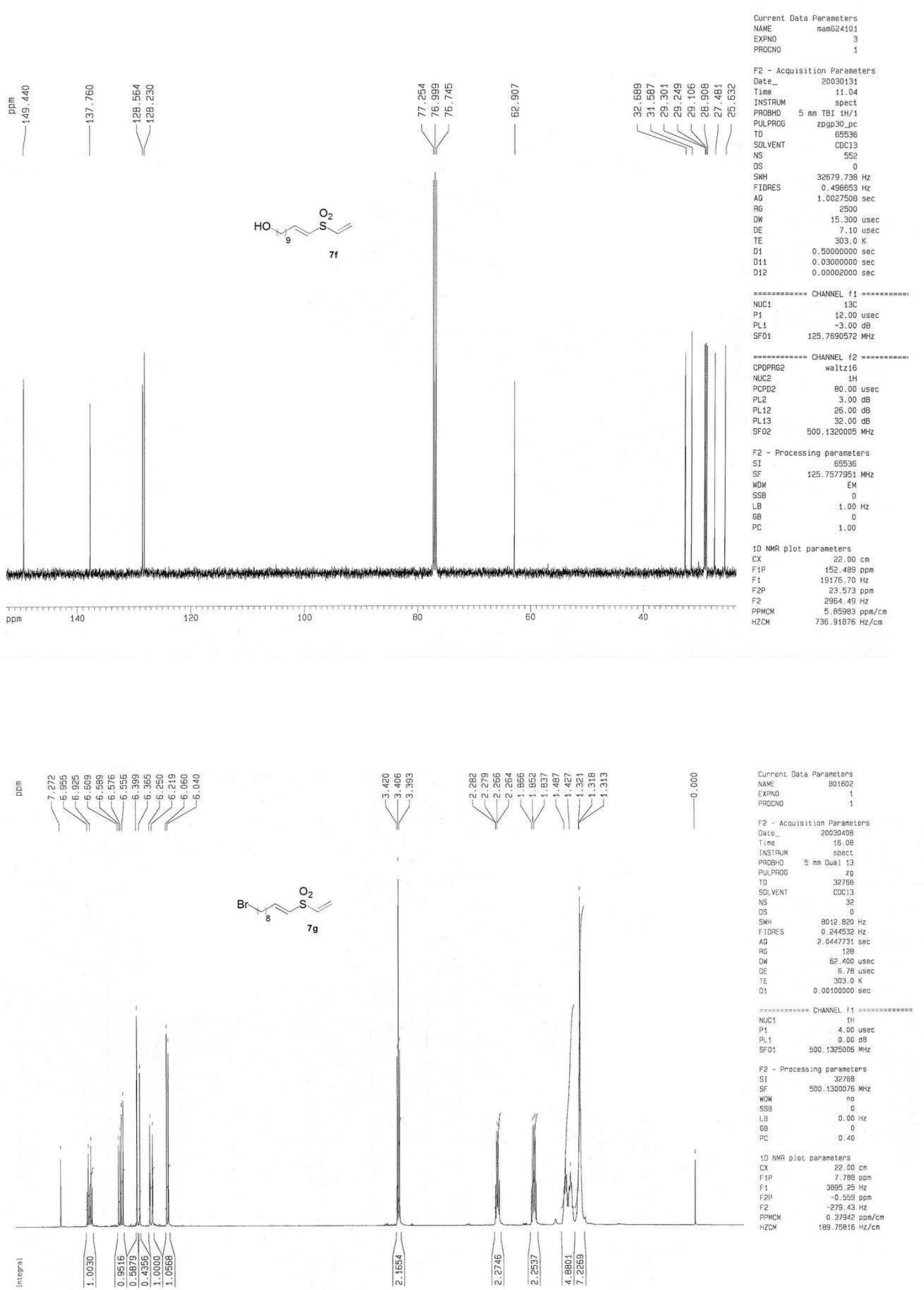

ppm 1+1 7
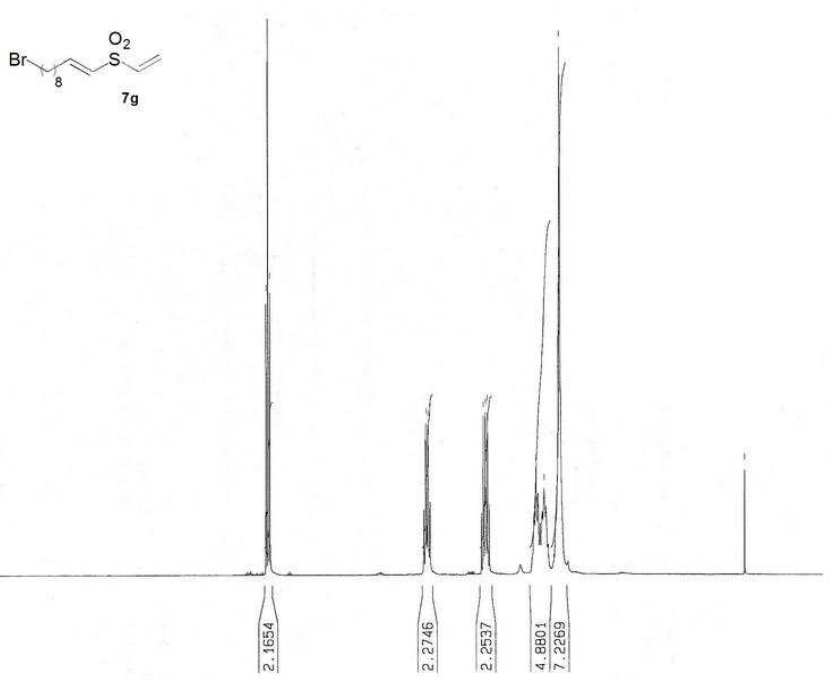

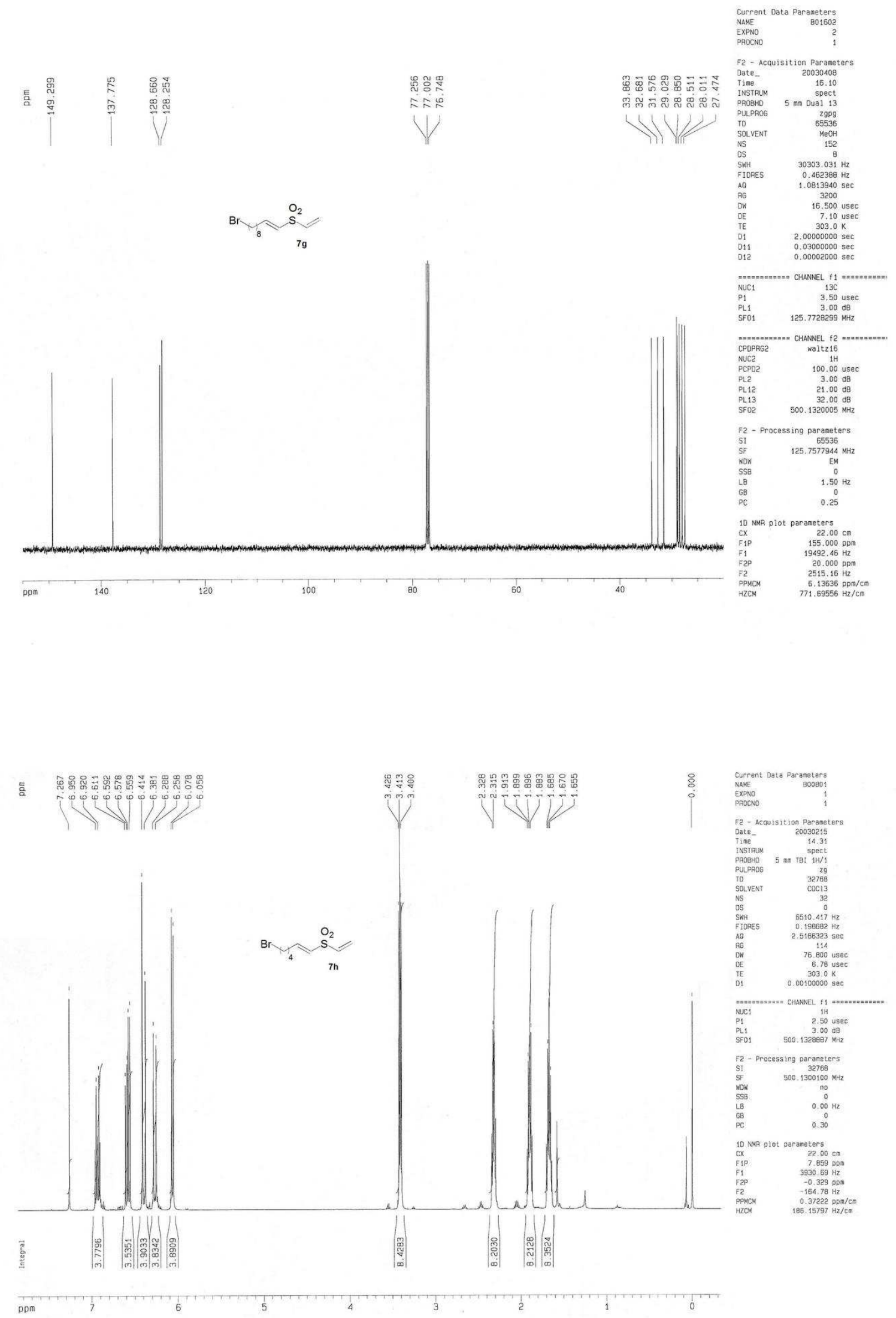

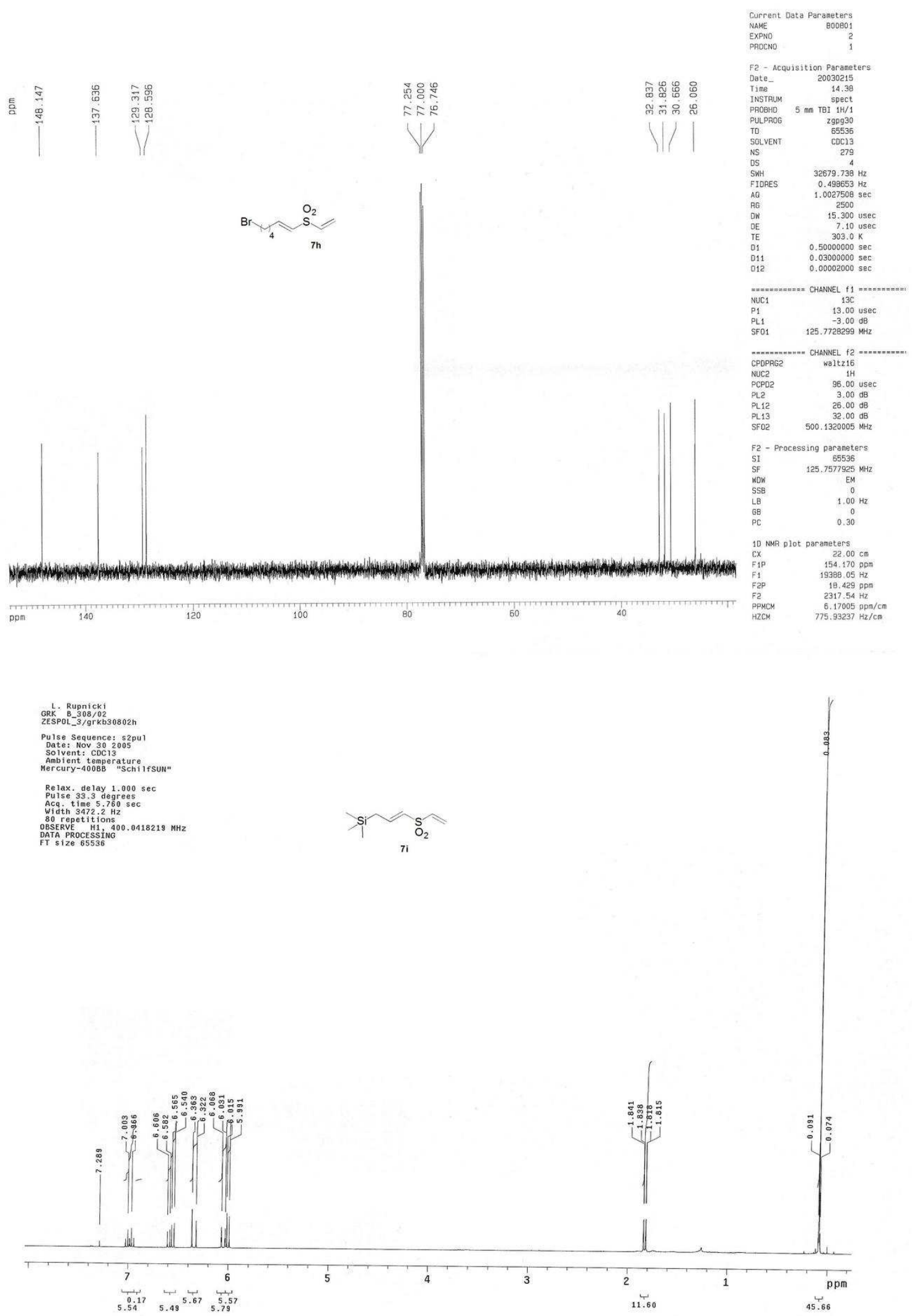
Dialkenyl sulfones via cross-metathesis of divinyl sulfone

Bieniek at al.
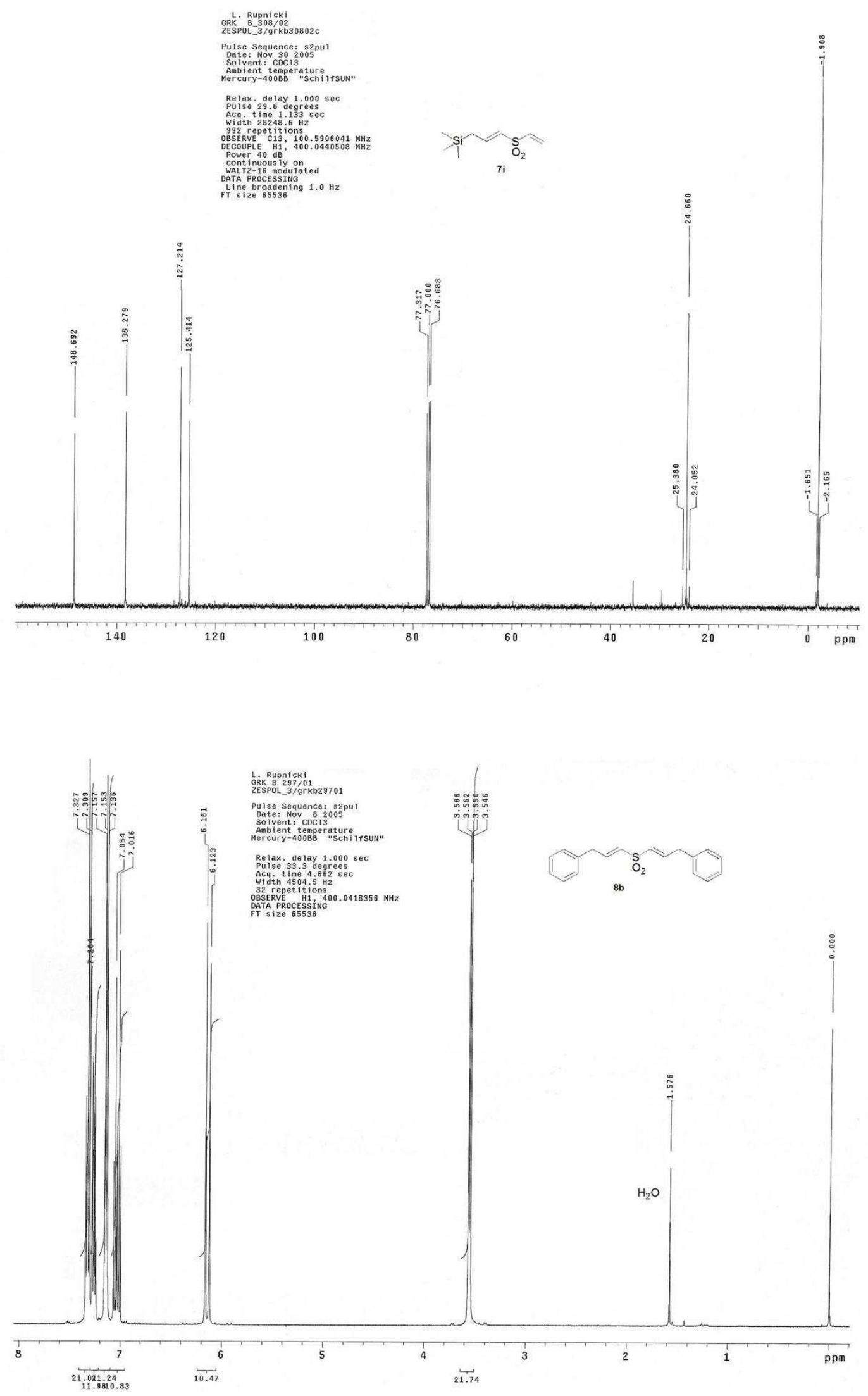

Supplementary Information

S -14 

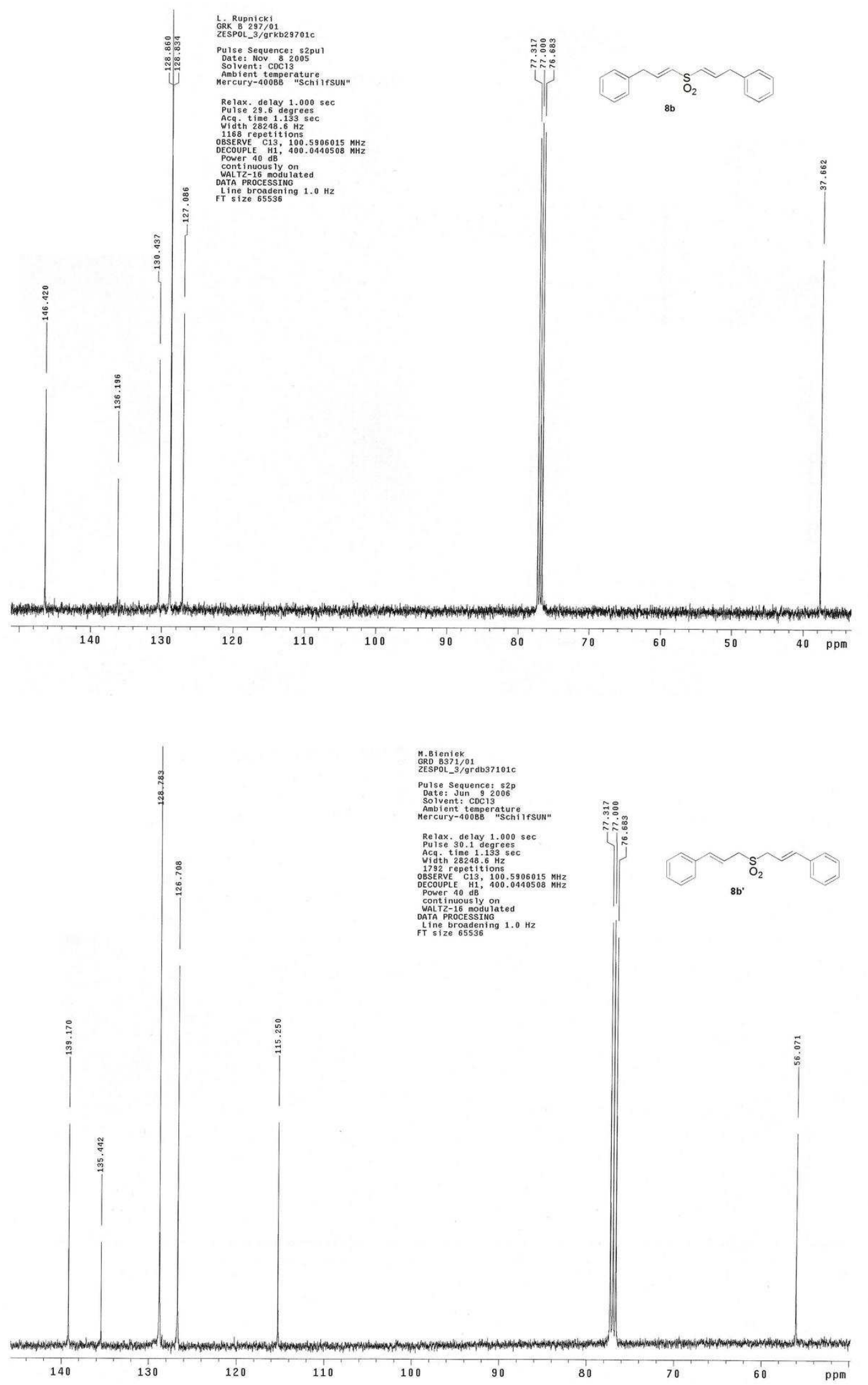

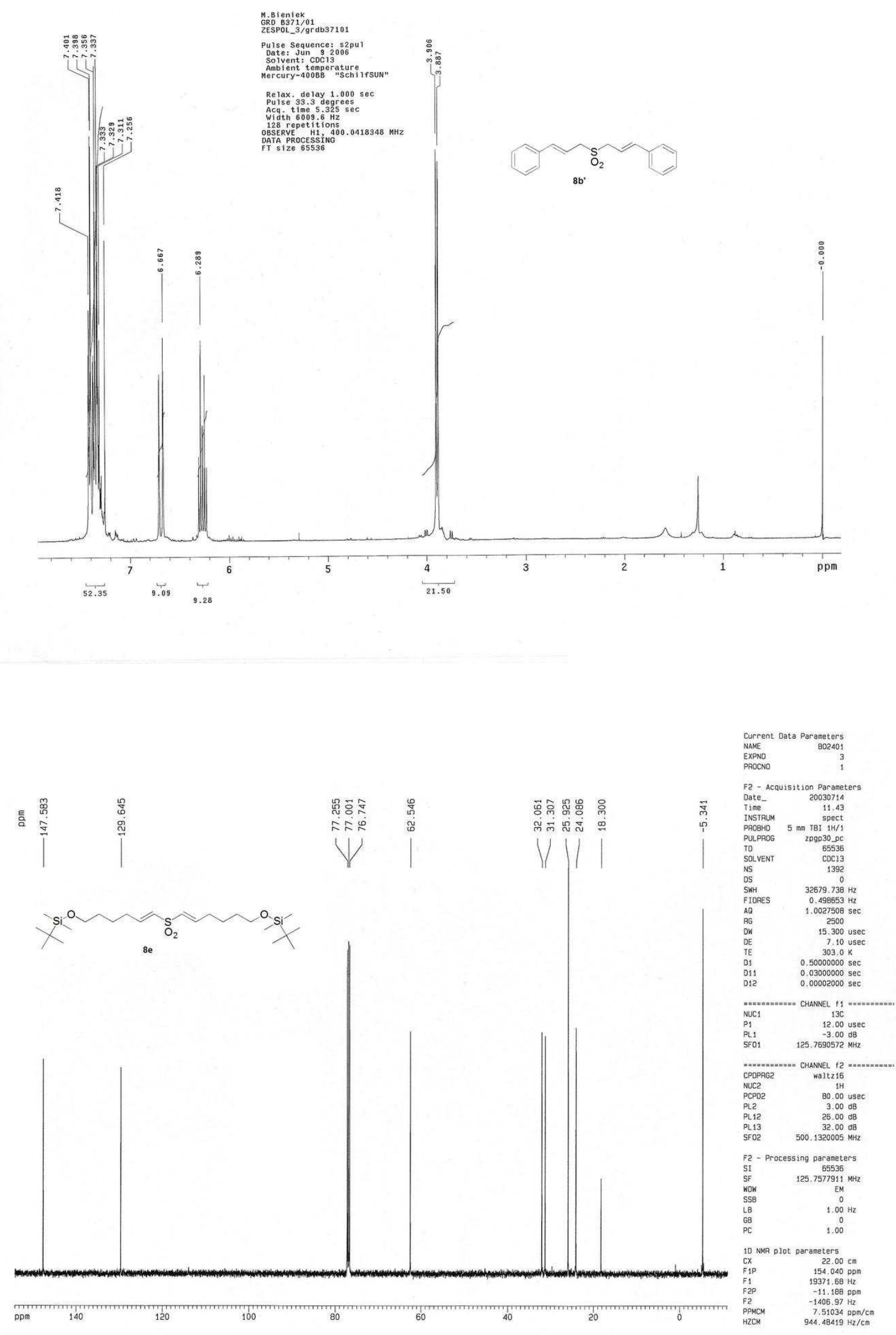

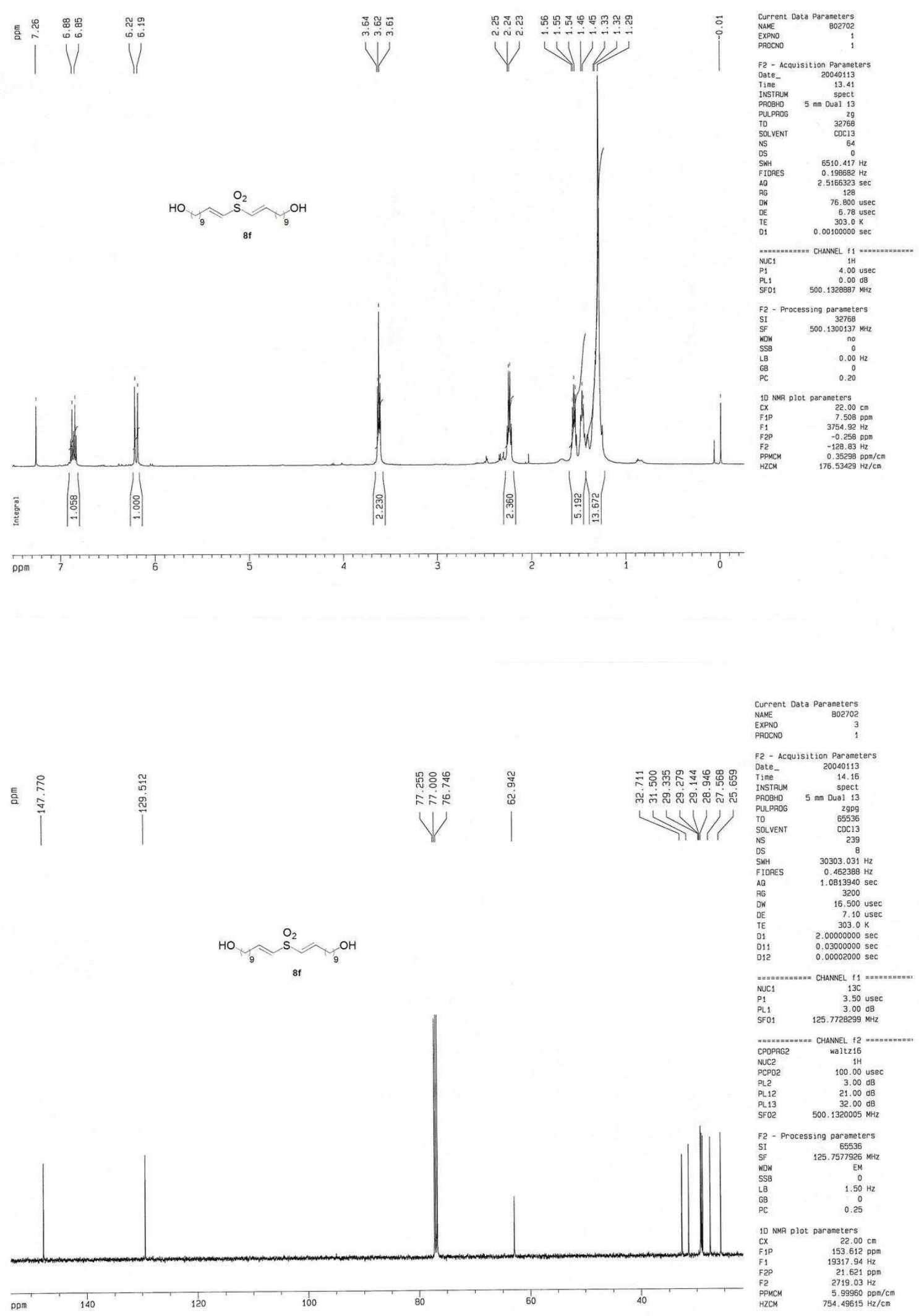

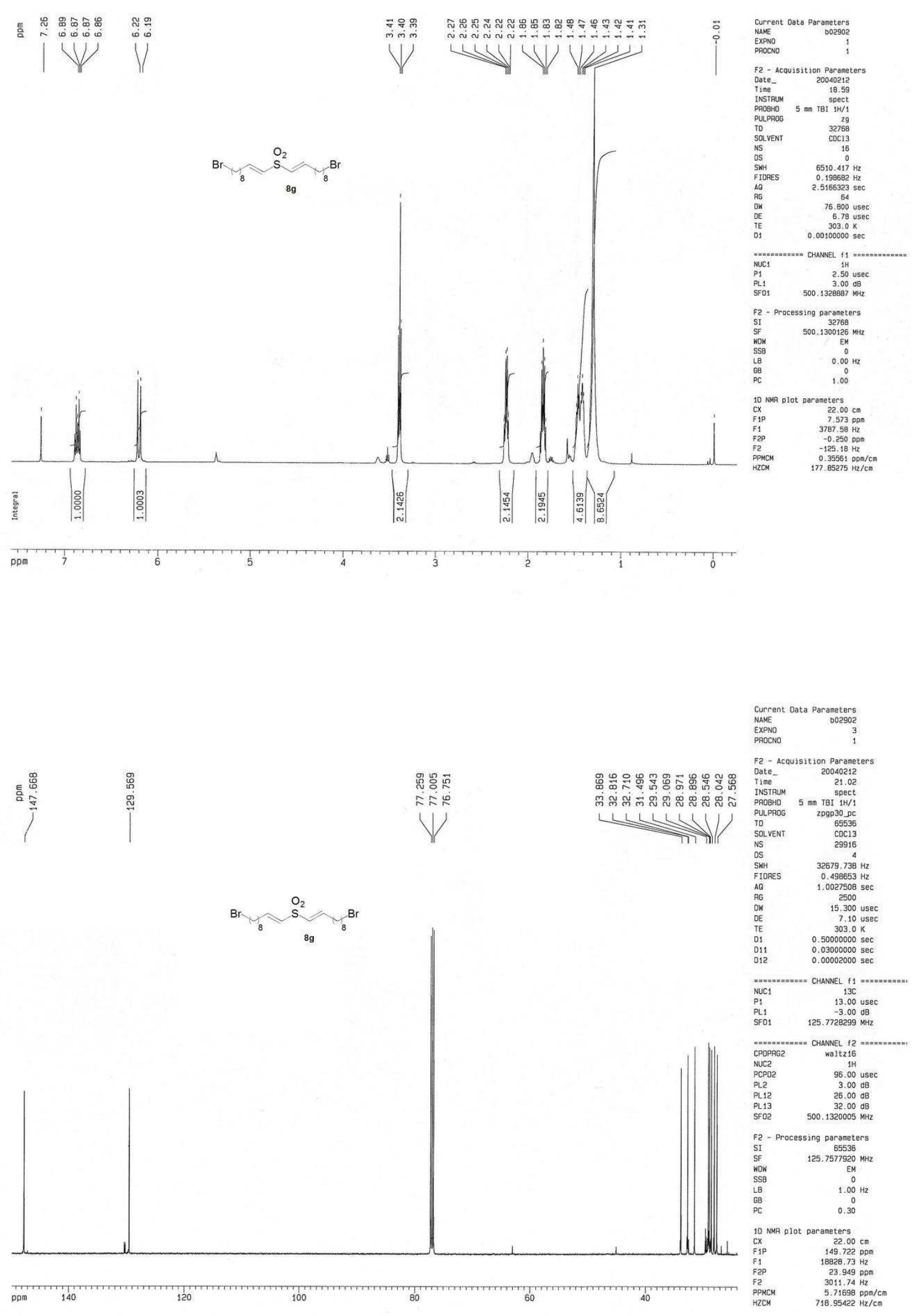

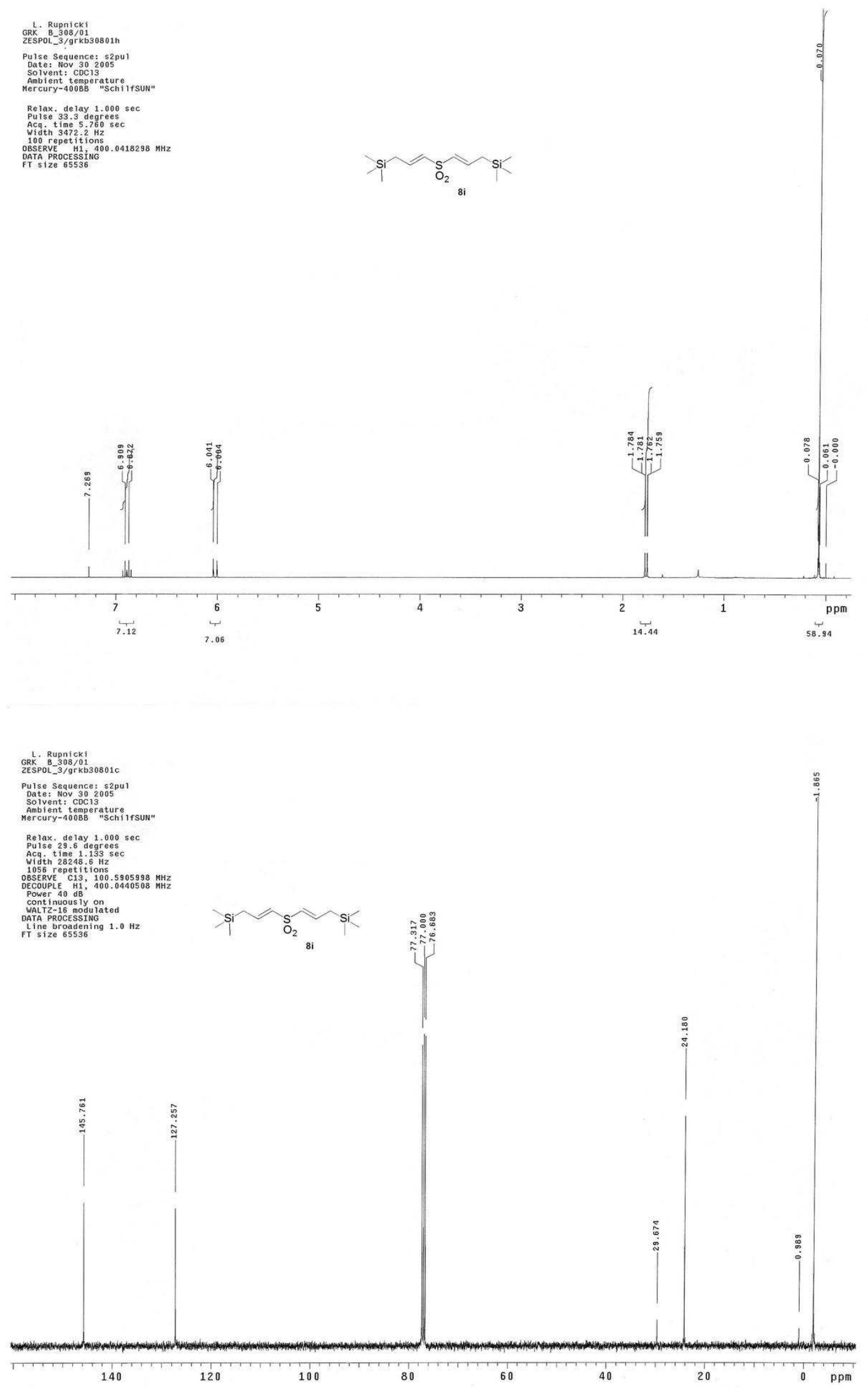

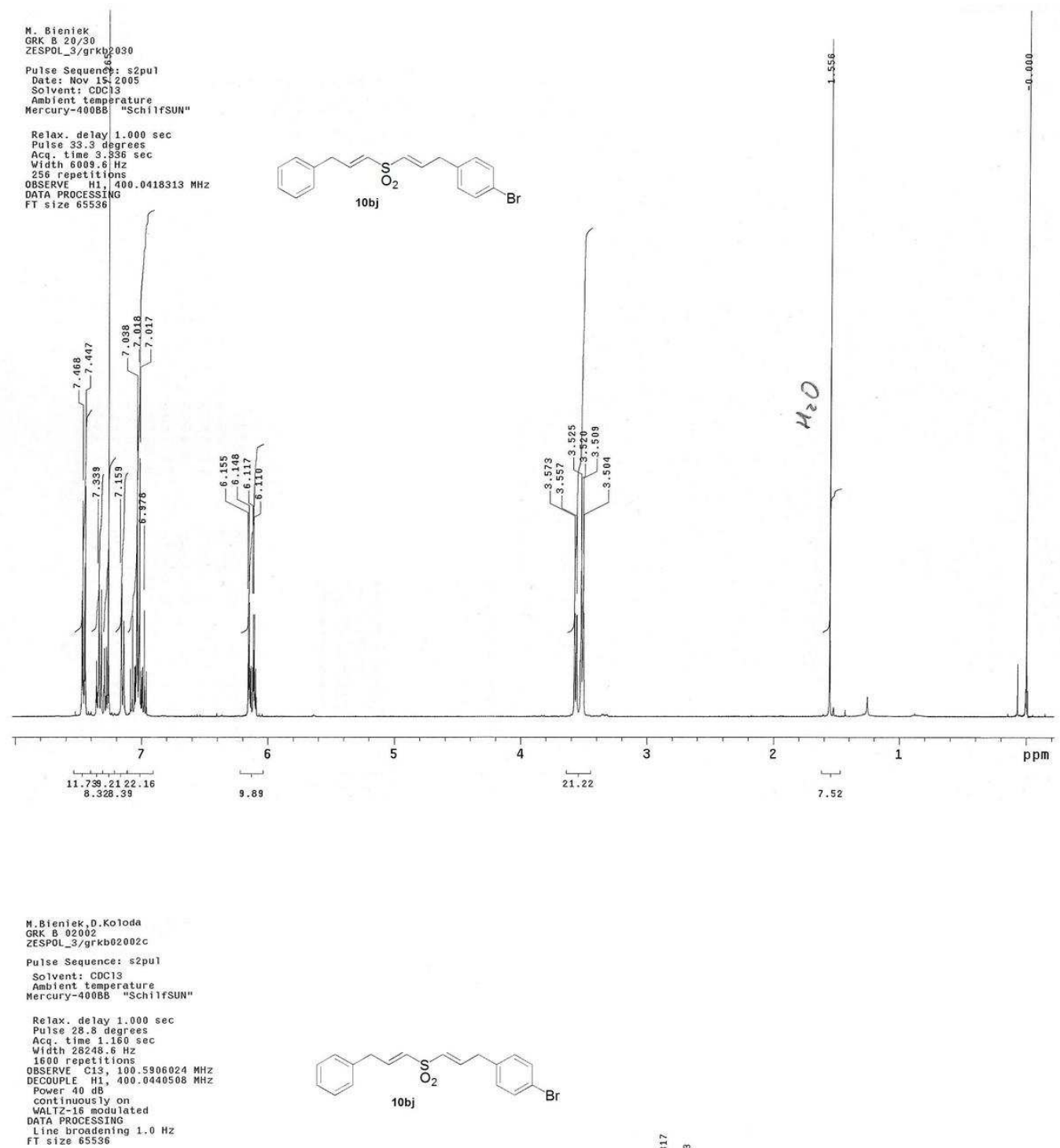

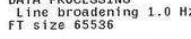

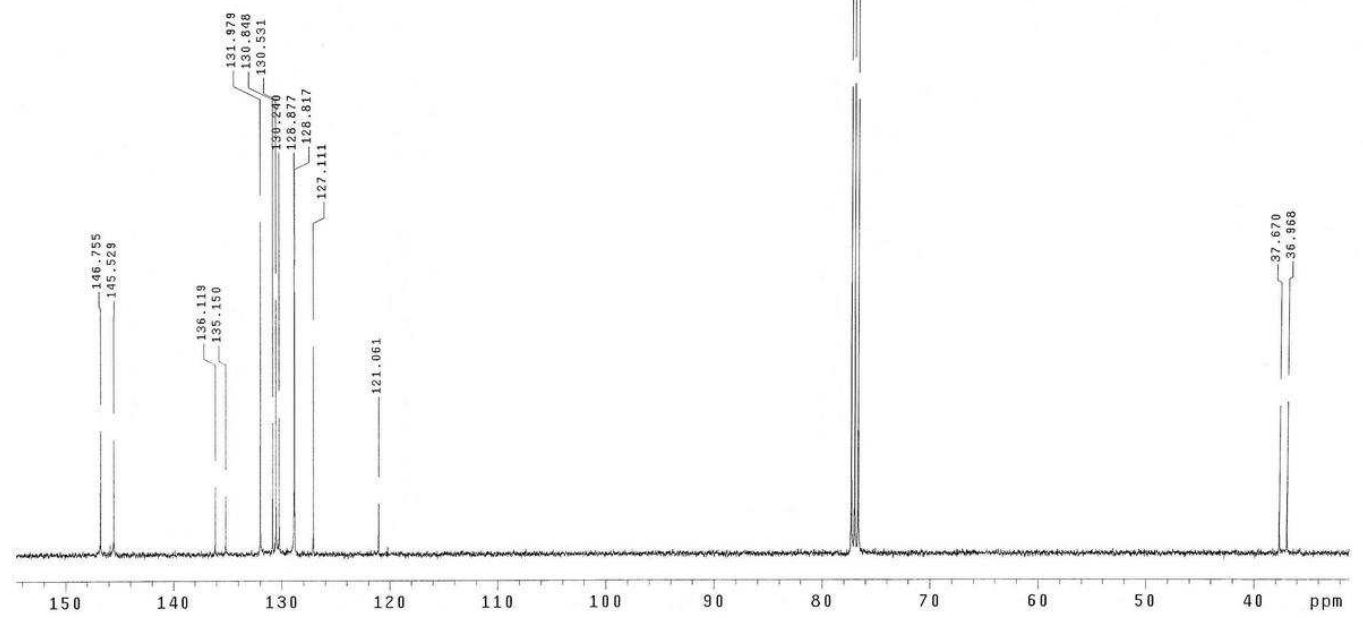



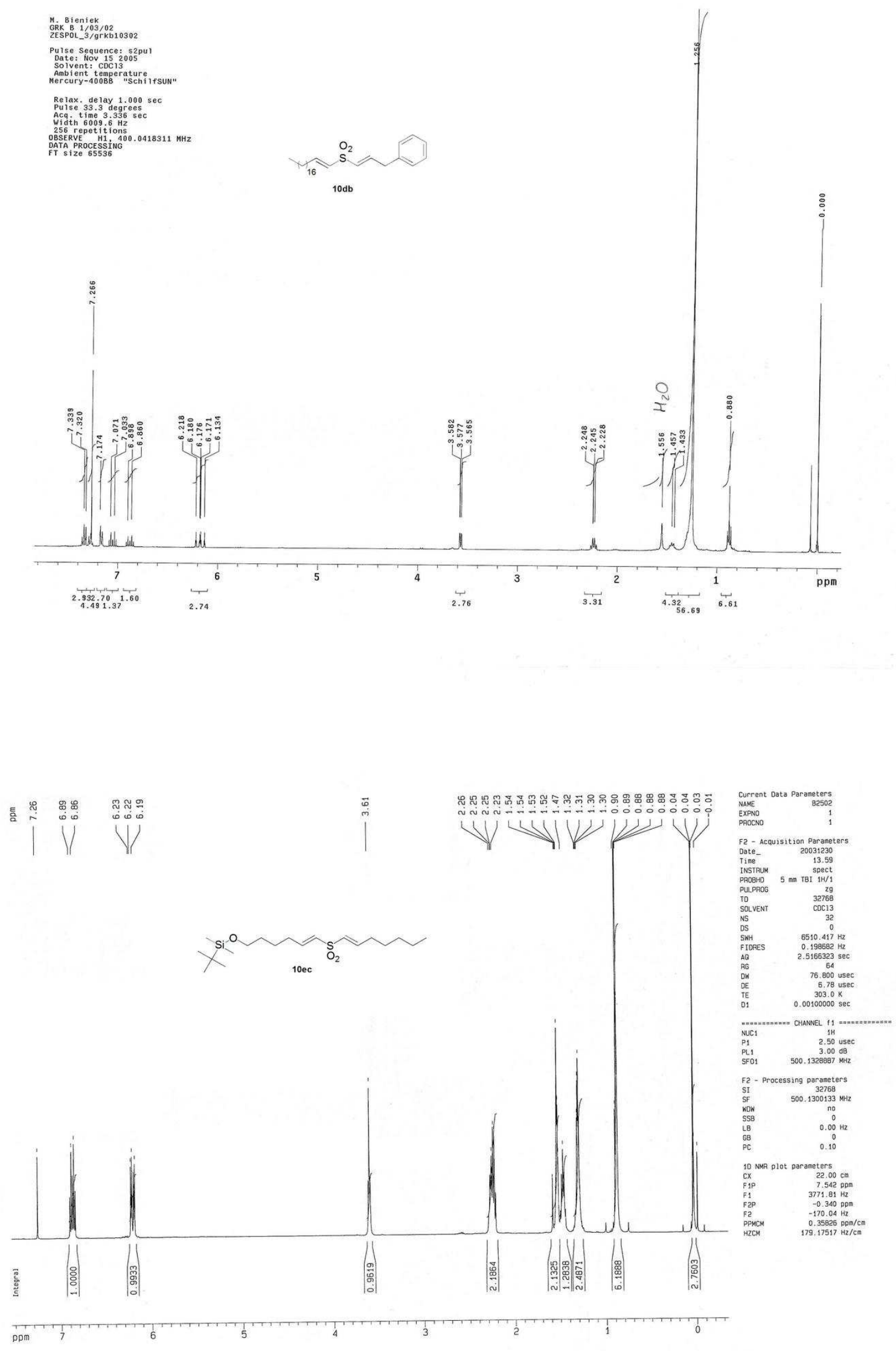

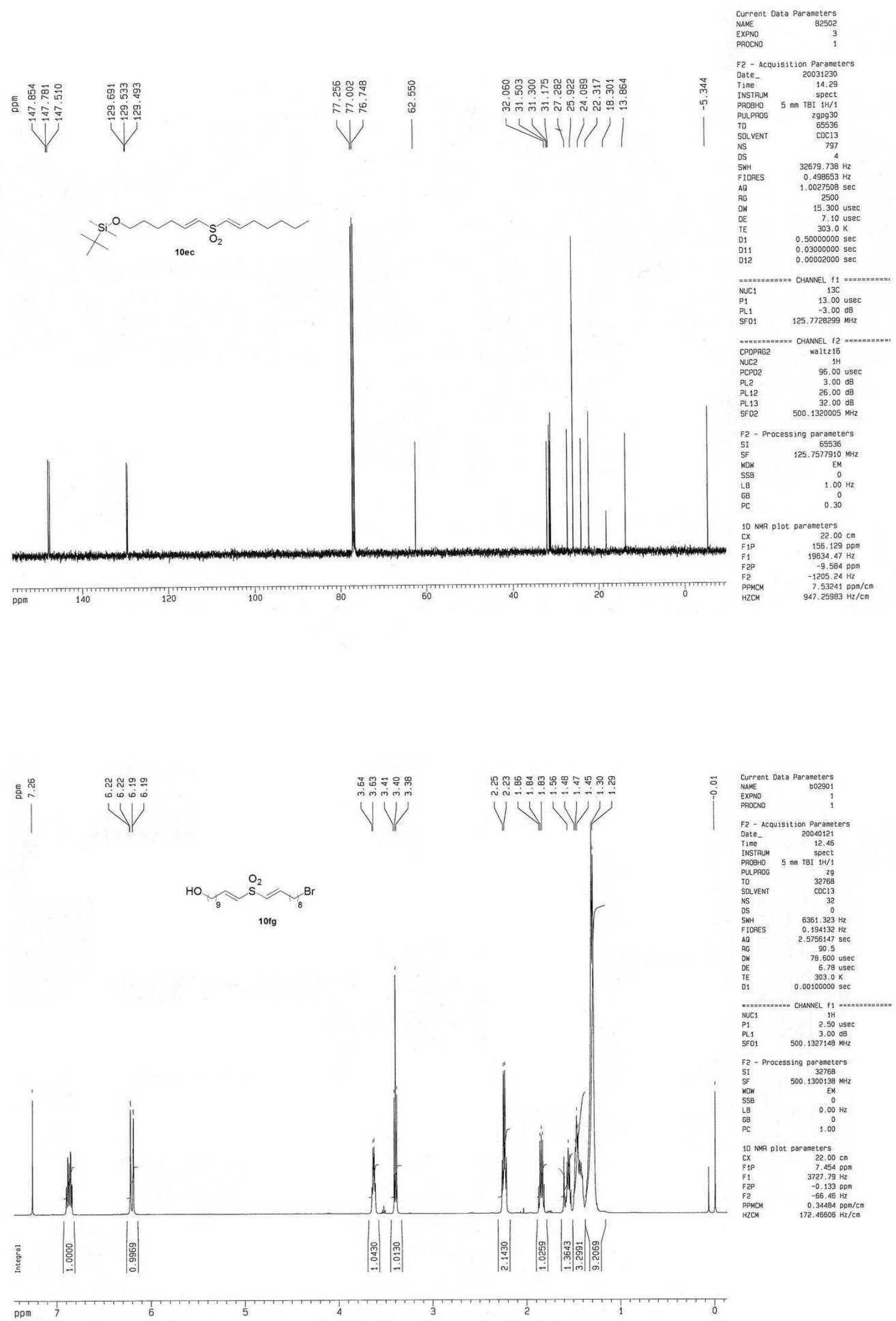

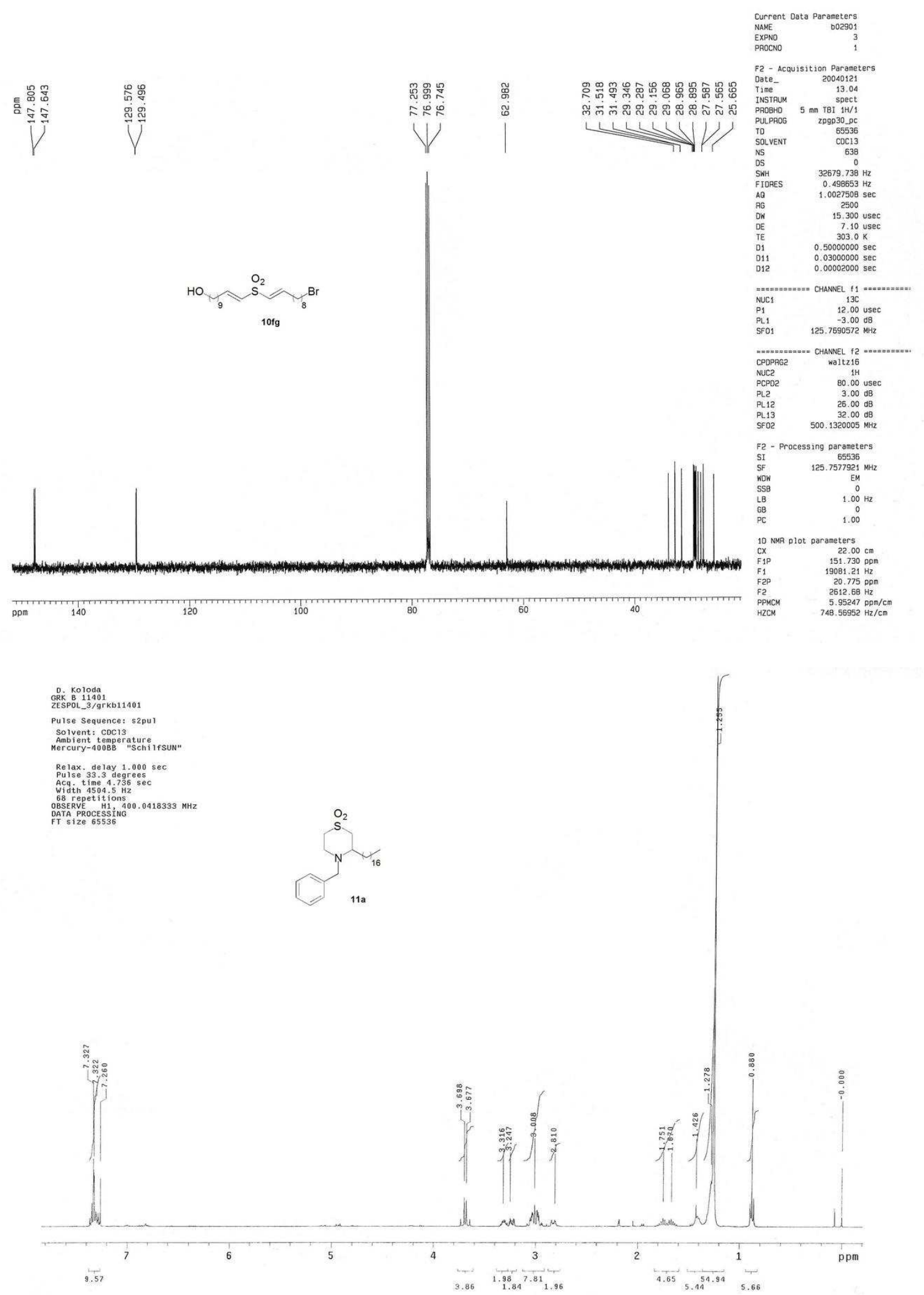

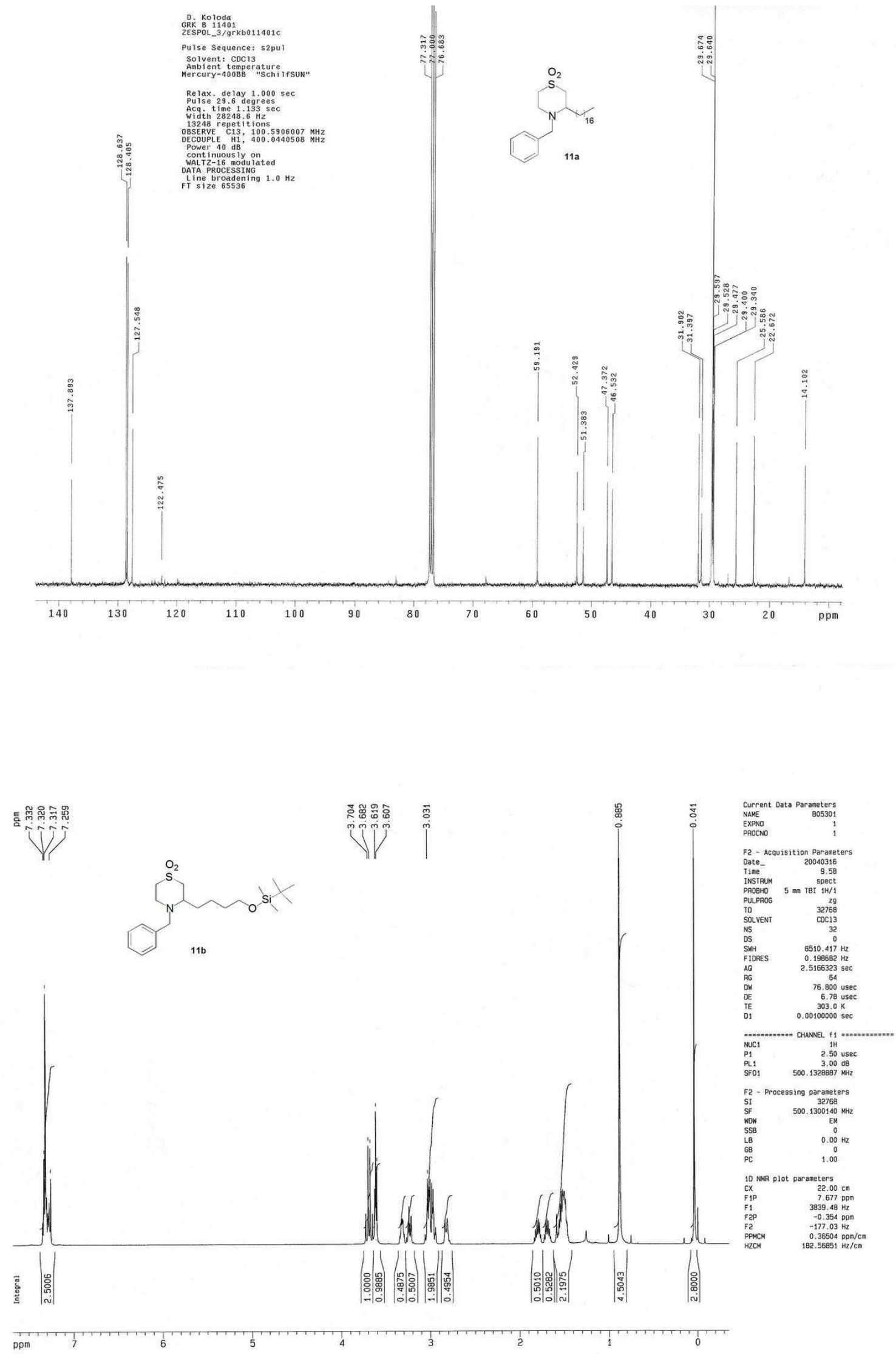

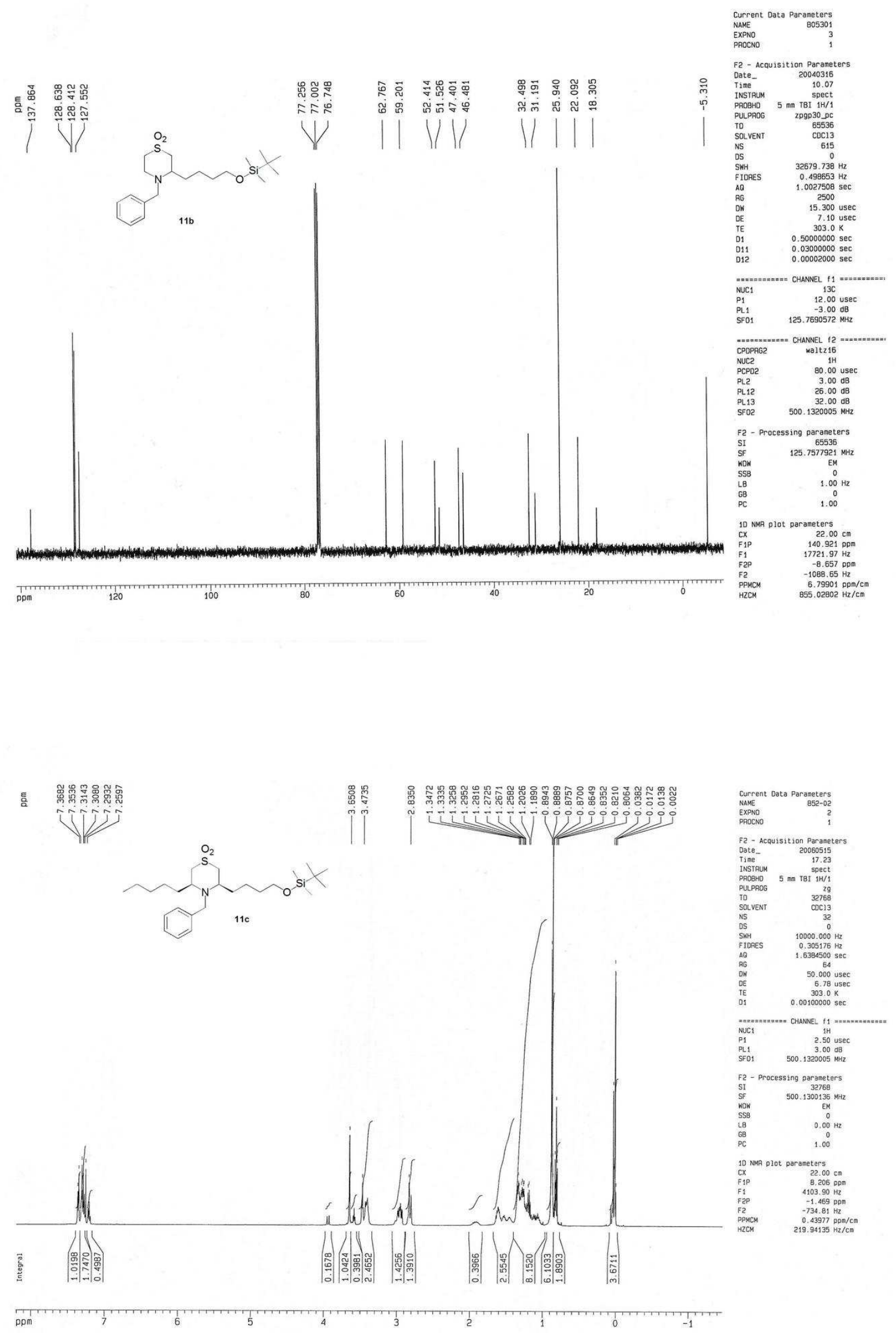

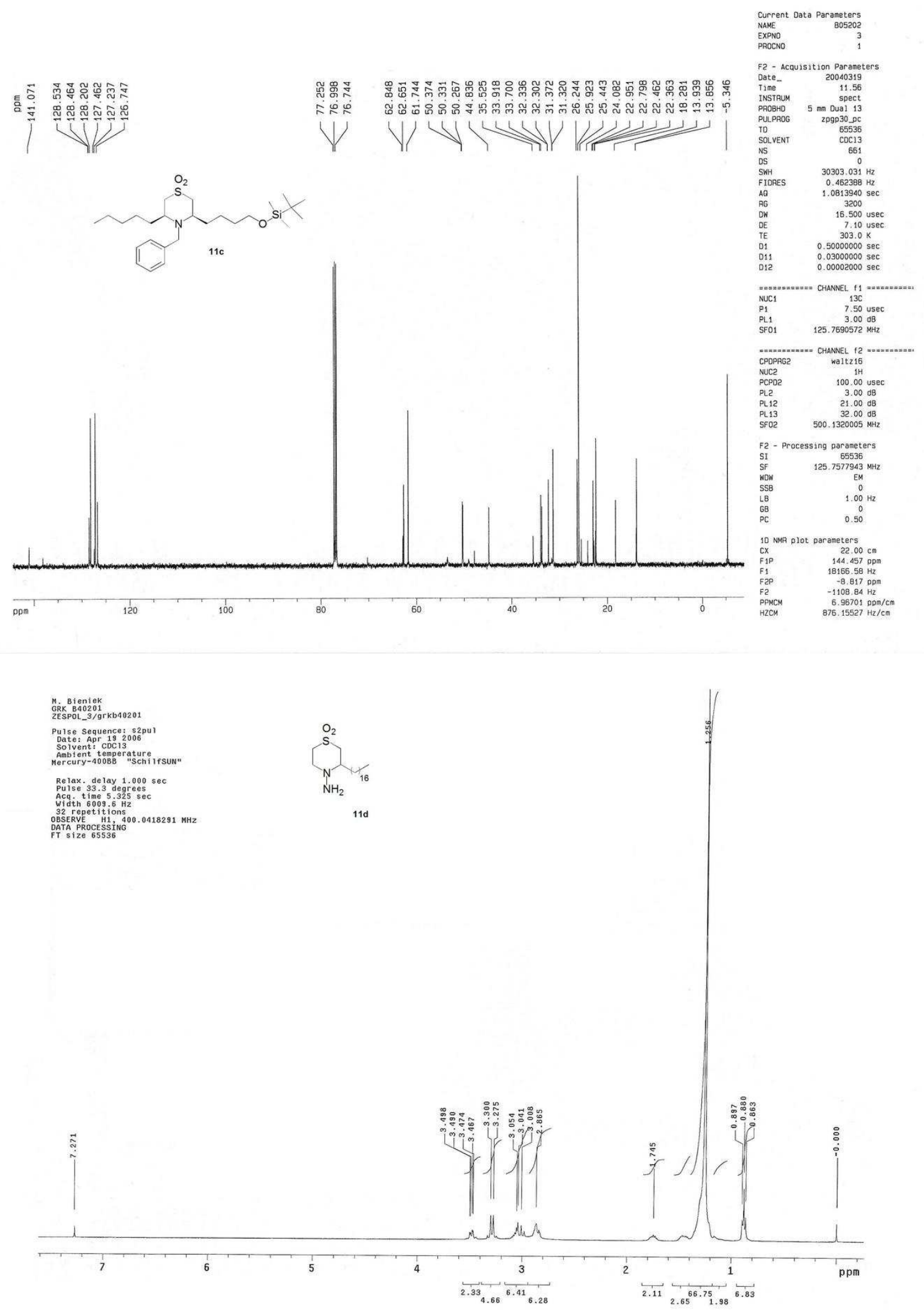

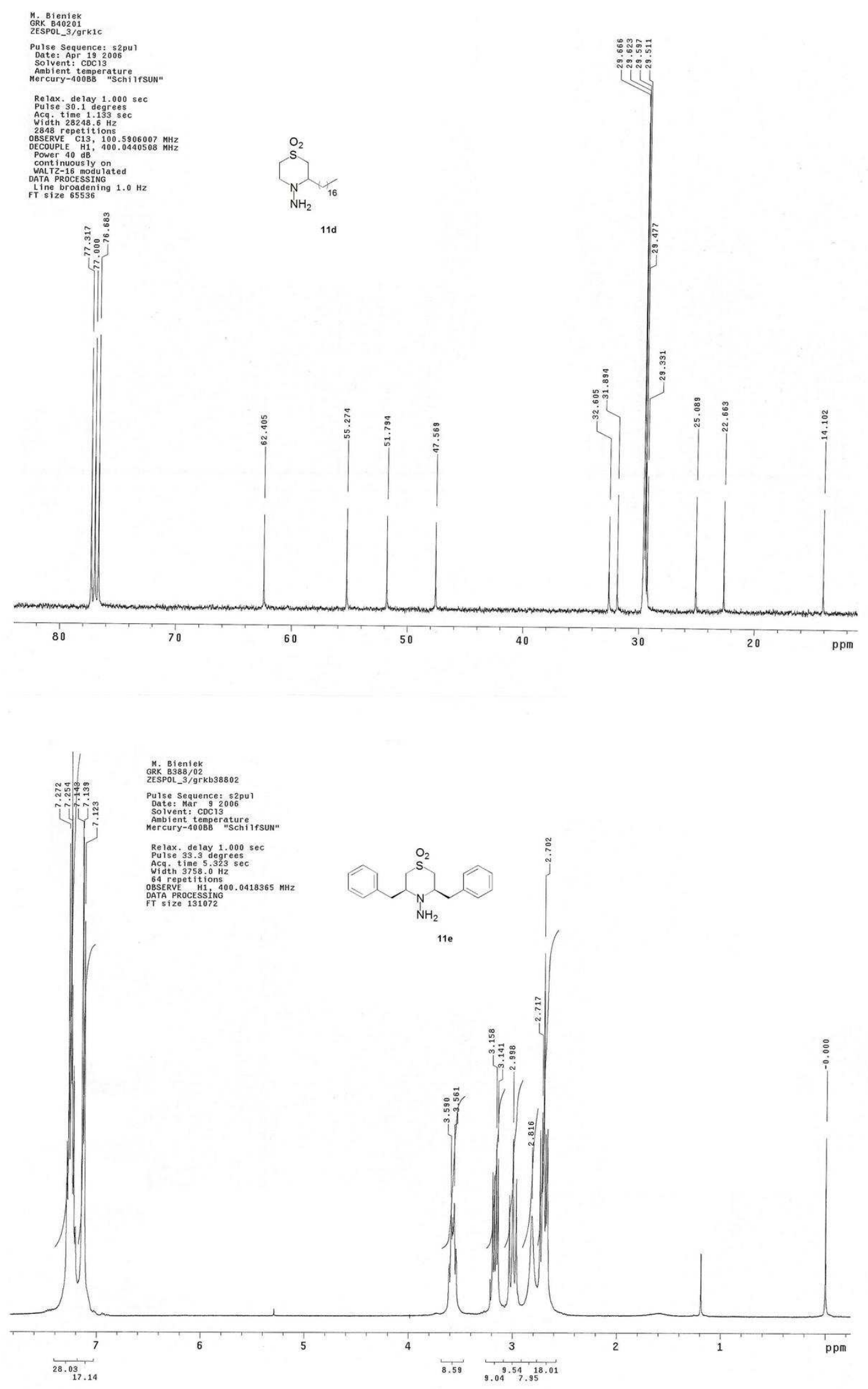

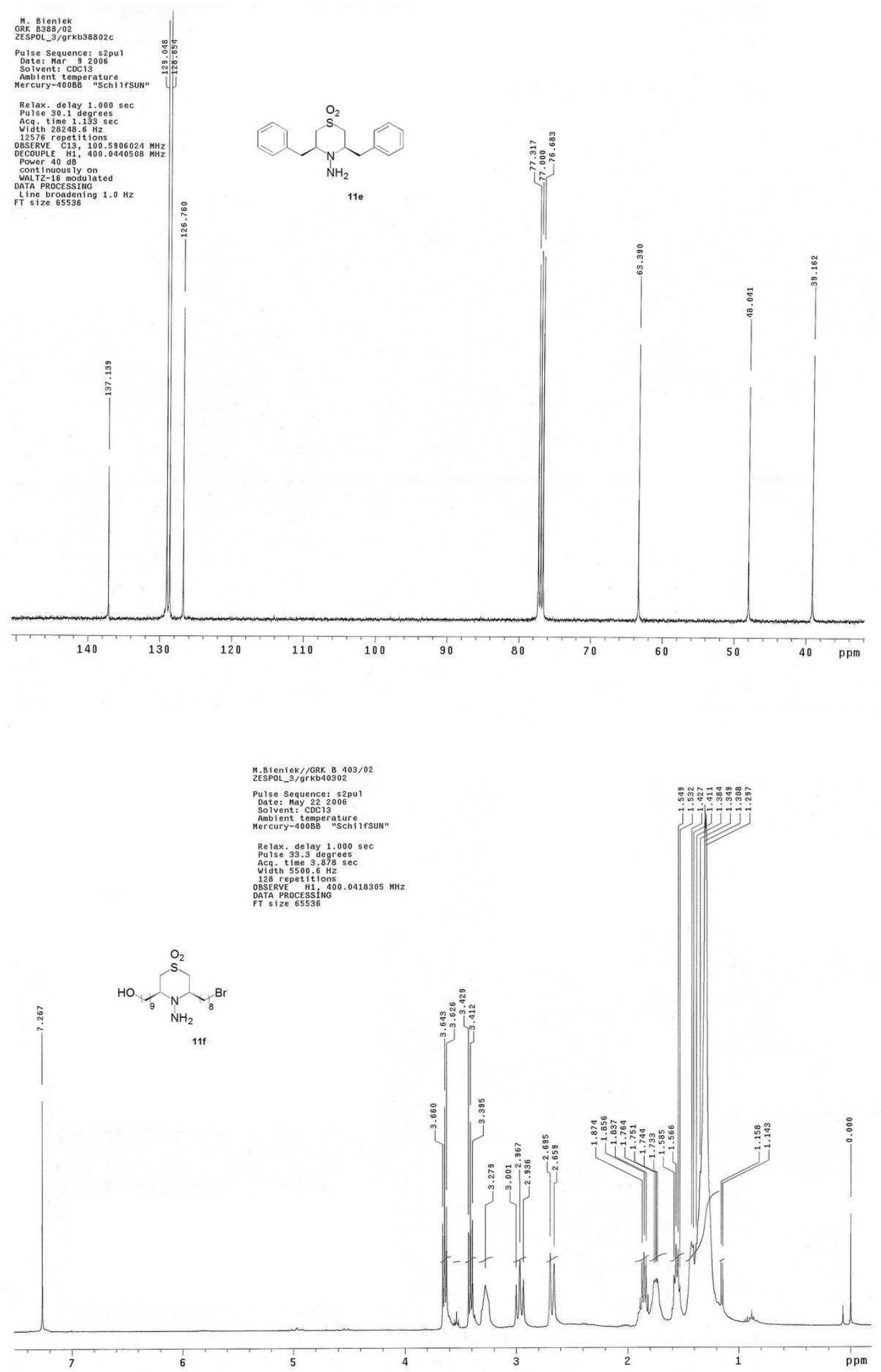


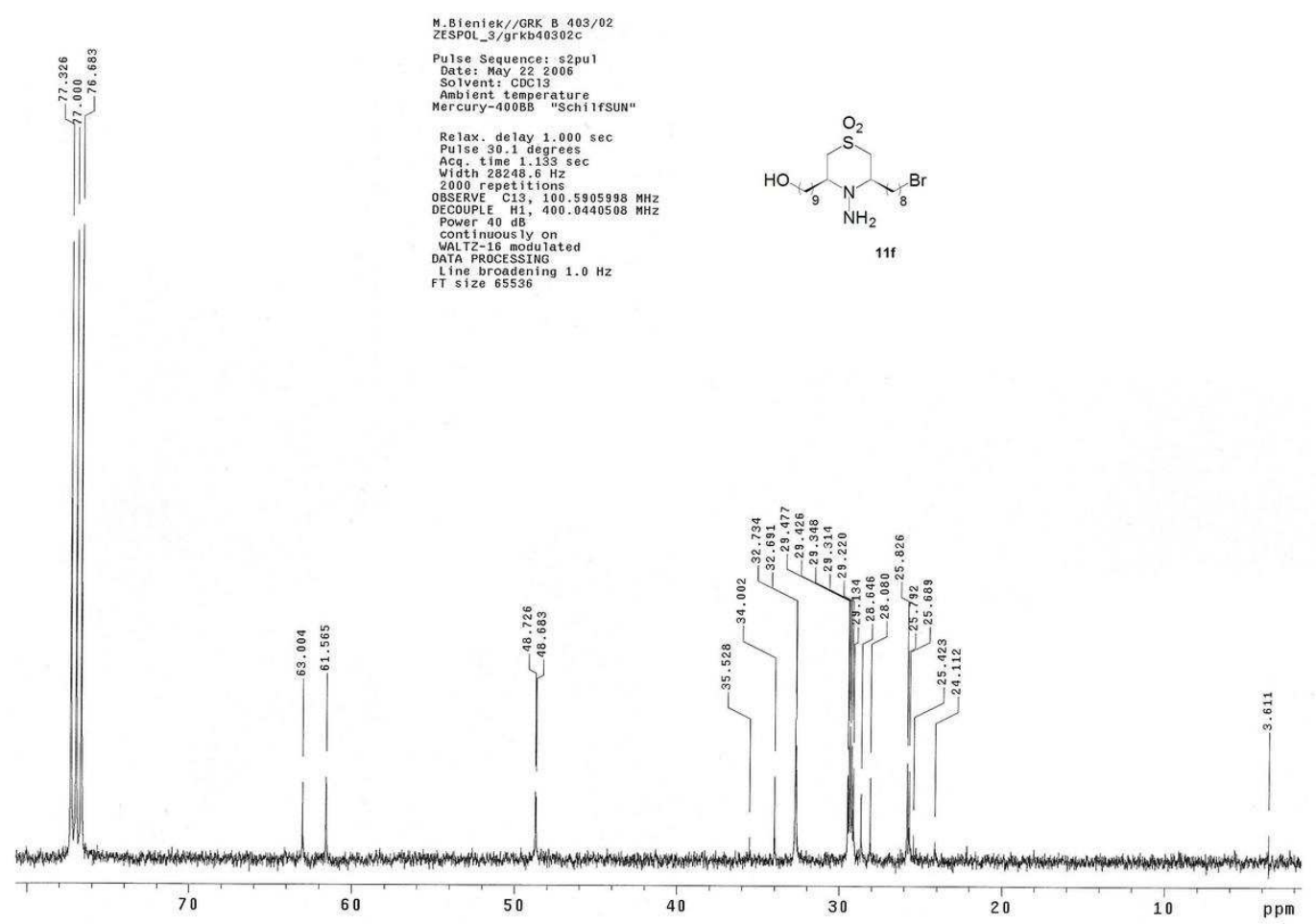

